Document downloaded from:

http://hdl.handle.net/10251/120344

This paper must be cited as:

Vázquez-Lozano, JE.; Cordero Barbero, A.; Torregrosa Sánchez, JR. (2018). Dynamical analysis on cubic polynomials of Damped Traub s method for approximating multiple roots. Applied Mathematics and Computation. 328:82-99.

https://doi.org/10.1016/j.amc.2018.01.043

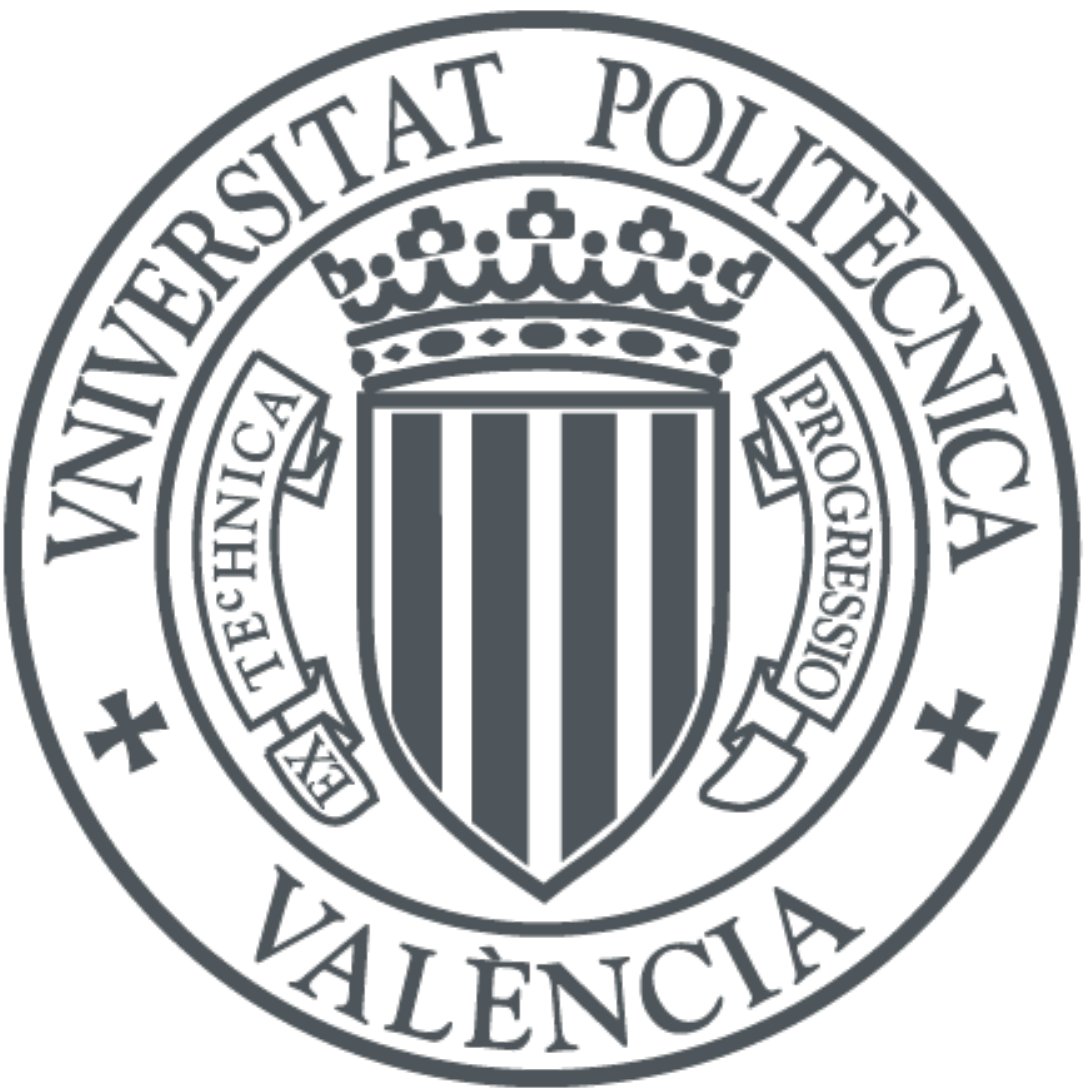

The final publication is available at

http://doi.org/10.1016/j.amc.2018.01.043

Copyright Elsevier

Additional Information 


\title{
Dynamical analysis on cubic polynomials of Damped Traub's method for approximating multiple roots और
}

\author{
J. Enrique Vázquez-Lozano ${ }^{\mathrm{a}}$, Alicia Cordero ${ }^{\mathrm{b}, *}$, Juan R. Torregrosa ${ }^{\mathrm{b}}$ \\ ${ }^{a}$ Nanophotonics Technology Center, Universitat Politècnica de València, Camino de Vera s/n, 46022 València, Spain \\ ${ }^{b}$ Instituto de Matemáticas Multidisciplinar, Universitat Politècnica de València, Camino de Vera s/n, 46022 València, Spain
}

\begin{abstract}
In this paper, the performance of a parametric family including Newton's and Traub's schemes on multiple roots is analyzed. The local order of convergence on nonlinear equations with multiple roots is studied as well as the dynamical behavior in terms of the damping parameter on cubic polynomials with multiple roots. The fixed and critical points, and the associated parameter plane are some of the characteristic dynamical features of the family which are obtained in this work. From the analysis of these elements we identify members of the family of methods with good numerical properties in terms of stability and efficiency both for finding the simple and multiple roots, and also other ones with very unstable behavior.
\end{abstract}

Keywords: Nonlinear equations; Iterative methods; Multiple roots; Complex dynamics; Convergence regions; Parameter space; Fixed point; Critical point

\section{Introduction}

Solving nonlinear equations is an important problem with many applications in Science and Engineering. In this study, we consider nonlinear equations $f(x)=0$, where $f: I \subseteq \mathbb{R} \rightarrow \mathbb{R}$ is a scalar function defined on an open interval $I$. For solving these equations, iterative schemes must be used. One of the most known and basic iterative method is the one-point Newton's scheme. It converges quadratically for simple roots if the initial guess $x_{0}$ is chosen close enough to the required root. In the last decades, many researchers have presented different methods to improve the order and the stability of Newton's scheme (see, for example, the overview that appears in [1] and [2] and references therein).

A common way to generate new schemes is the direct composition of known methods with a later treatment to reduce the number of functional evaluations: for example, by composing Newton's method with itself, holding the derivative "frozen" in the second step, third-order Traub's method [3] is obtained.

In [4], the authors presented a damped Traub's family of iterative methods, whose iterative expression is

$$
z_{n+1}=y_{n}-\gamma \frac{f\left(y_{n}\right)}{f^{\prime}\left(z_{n}\right)}, \quad n=0,1, \ldots,
$$

where $y_{n}=z_{n}-\frac{f\left(z_{n}\right)}{f^{\prime}\left(z_{n}\right)}$ is a Newton's step and $\gamma$ is the damping parameter. Let us note that, if $\gamma=1$ we get the Traub's scheme and $\gamma=0$ leads Newton's method. The order of convergence of family (1) on nonlinear equations with multiple roots is studied in this work in terms of the $\gamma$ parameter.

The application of iterative methods for solving nonlinear equations on polynomials gives rise to rational functions whose complex dynamics leads to valuable information about their dependence on initial estimations. However, Amat et al. in [5] studied real dynamics of Traub's scheme on quadratic and cubic polynomials. From the numerical point of view, the dynamical behavior of the rational function associated with an iterative method gives us important information about its stability and reliability. In these terms, Amat et al. in [6] described the dynamical behavior of several well-known families of iterative methods. More recently, in [7, 8, 9, 10, 11, 12], the authors analyze the qualitative behavior of different known iterative families. Most of these studies show different pathological numerical behavior, such as periodic

\footnotetext{
This research was partially supported by Ministerio de Economía y Competitividad MTM2014-52016-C2-2-P and Generalitat Valenciana PROMETEO/2016/089.

* Corresponding author

Email addresses: juavazlo@ntc.upv.es (J. Enrique Vázquez-Lozano), acordero@mat.upv.es (Alicia Cordero ), jrtorre@mat.upv.es (Juan R. Torregrosa )
} 
orbits, attracting fixed points different from the solution of the problem, among others. Therefore, from the analysis of the parameter planes associated to a family of methods we will gain understanding about the behavior of the different members of the family of methods, helping us in the election of a particular one.

Very recently, in $[13,14,15,16]$ the dynamical behavior of iterative methods for solving nonlinear equations with multiple roots is analyzed. The use of complex dynamics tools on these kind of problems is very fruitful, as their stability properties show. Very often, the stability region of multiple roots is wide but the respective one of the simple root is extremely small or even does not exist.

In this paper, we describe the dynamical behavior of family (1) on cubic polynomials with multiple roots. Specifically, we analyze the stability of the family on $p(z)=(z-a)^{2}(z-b)$, obtaining interesting information about the dynamics of (1), distinguishing between the multiple root and the simple one.

The rest of the paper is organized as follows: in Section 2 we study the local convergence of family (1) for simple and multiple roots. Section 3 is devoted to introduce the basic concepts on complex dynamics, which is used in Section 4, where the analysis of the fixed and critical points of the rational operator associated to the family on $p(z)$ is showed. The regions of stability (Section 5) also appear in the associated parameter spaces (Section 6), as well as the stability region of the attractive 2-periodic orbits. In order to illustrate the previous results, in Section 7 we plot some examples of the dynamical planes showing both the stable and unstable behavior. We finish the paper with some remarks and conclusion that extend the earlier results in this fruitful area of research.

\section{Analysis of convergence}

In the following result we show the local convergence of the Traub's family of iterative methods (1) when it is applied to a nonlinear equation with simple or multiple roots of arbitrary multiplicity $m \geq 1$.

Theorem 1. Let $f: D \subseteq \mathbb{R} \longrightarrow \mathbb{R}$ be a sufficiently differentiable function in an open interval $D$. We assume that $f(z)=0$ has a multiple root $\alpha$ of multiplicity $m \geq 1$ in $D$. If $z_{0}$ is close enough to $\alpha$, then the members of the family (1) converge to $\alpha$ satisfying the following error equation:

$$
e_{n+1}=\mathcal{E}_{m}^{(1)}(\gamma) e_{n}+\mathcal{E}_{m}^{(2)}(\gamma) e_{n}^{2}+\mathcal{E}_{m}^{(3)}(\gamma) e_{n}^{3}+\mathcal{O}\left[e_{n}^{4}\right]
$$

where

$$
\begin{aligned}
& \mathcal{E}_{m}^{(1)}(\gamma)=1-m^{-(m+1)}\left[m^{m}+\gamma(m-1)^{m}\right] \\
& \mathcal{E}_{m}^{(2)}(\gamma)=\frac{c_{m+1}\left[\gamma\left(m^{2}-m-1\right) A(m)+1\right]}{m^{2}(m+1)} ; \\
& \mathcal{E}_{m}^{(3)}(\gamma)=\frac{2(m+1)\left[2 m c_{m+2}-(m+2) c_{m+1}^{2}\right]+\gamma A(m)\left[2 m(m-2)(m+1)^{2} c_{m+2}-(m+2)\left(2 m^{3}-2 m^{2}-5 m-2\right) c_{m+1}^{2}\right]}{2 m^{3}(1+m)^{2}(m+2)}, \\
& \text { and } A(m)=\left(\frac{(m-1)^{m-1}}{m^{m}}\right) \text { and } e_{n}=z_{n}-\alpha, c_{j}=f^{(j)}(\alpha) / f^{(m)}(\alpha), j \geq m+1 .
\end{aligned}
$$

Proof. Let $\alpha$ be a root of multiplicity $m$ (i.e. $f(\alpha)=f^{\prime}(\alpha)=f^{\prime \prime}(\alpha)=\ldots=f^{(m-1)}(\alpha)=0$ and $\left.f^{(m)}(\alpha) \neq 0\right)$. By using Taylor expansion of $f\left(z_{n}\right)$ around $\alpha$ we get

$$
\begin{aligned}
f\left(z_{n}\right) & =f(\alpha)+f^{\prime}(\alpha) e_{n}+\frac{f^{(2)}(\alpha)}{2 !} e_{n}^{2}+\ldots+\frac{f^{(m)}(\alpha)}{m !} e_{n}^{m}+\ldots+\mathcal{O}\left[e_{n}^{m+3}\right] \\
& =\frac{f^{(m)}(\alpha)}{m !} e_{n}^{m}\left\{1+\frac{c_{m+1}}{(m+1)} e_{n}+\frac{c_{m+2}}{(m+2)(m+1)} e_{n}^{2}+\mathcal{O}\left[e_{n}^{3}\right]\right\} .
\end{aligned}
$$

Similarly, we obtain

$$
\begin{aligned}
f^{\prime}\left(z_{n}\right) & =f^{\prime}(\alpha)+f^{(2)}(\alpha) e_{n}+\frac{f^{(3)}(\alpha)}{2 !} e_{n}^{2}+\ldots+\frac{f^{(m)}(\alpha)}{(m-1) !} e_{n}^{m-1}+\ldots+\mathcal{O}\left[e_{n}^{m+2}\right] \\
& =\frac{f^{(m)}(\alpha)}{(m-1) !} e_{n}^{m-1}\left\{1+\frac{c_{m+1}}{m} e_{n}+\frac{c_{m+2}}{(m+1) m} e_{n}^{2}+\mathcal{O}\left[e_{n}^{3}\right]\right\} .
\end{aligned}
$$


By direct division of (2) and (3),

$$
\begin{aligned}
\frac{f\left(z_{n}\right)}{f^{\prime}\left(z_{n}\right)} & =\frac{e_{n}}{m}\left[\frac{1+\frac{c_{m+1}}{(m+1)} e_{n}+\frac{c_{m+2}}{(m+2)(m+1)} e_{n}^{2}+\mathcal{O}\left[e_{n}^{3}\right]}{1+\frac{c_{m+1}}{m} e_{n}+\frac{c_{m+2}}{(m+1) m} e_{n}^{2}+\mathcal{O}\left[e_{n}^{3}\right]}\right] \\
& =\frac{e_{n}}{m}-\frac{c_{m+1}}{m^{2}(m+1)} e_{n}^{2}+\frac{(2+m) c_{m+1}^{2}-2 m c_{m+2}}{m^{3}(m+1)(m+2)} e_{n}^{3}+\mathcal{O}\left[e_{n}^{4}\right] .
\end{aligned}
$$

Then, by (4)

$$
y_{n}=z_{n}-\frac{f\left(z_{n}\right)}{f^{\prime}\left(z_{n}\right)}=\alpha+\frac{m-1}{m} e_{n}+\frac{c_{m+1}}{m^{2}(m+1)} e_{n}^{2}-\frac{(2+m) c_{m+1}^{2}-2 m c_{m+2}}{m^{3}(m+1)(m+2)} e_{n}^{3}+\mathcal{O}\left[e_{n}^{4}\right] .
$$

Again, by (5) and Taylor's expansion around $\alpha$,

$$
\begin{aligned}
f\left(y_{n}\right) & =f(\alpha)+f^{\prime}(\alpha)\left[y_{n}-\alpha\right]+\ldots+\frac{f^{(m)}(\alpha)}{m !}\left[\left(y_{n}-\alpha\right)^{m}\right]+\mathcal{O}\left[\left(y_{n}-\alpha\right)^{m+1}\right] \\
& =\frac{f^{(m)}(\alpha)}{m !}\left[\frac{m-1}{m} e_{n}+\frac{c_{m+1}}{m^{2}(m+1)} e_{n}^{2}-\frac{(2+m) c_{m+1}^{2}-2 m c_{m+2}}{m^{3}(m+1)(m+2)} e_{n}^{3}+\mathcal{O}\left[e_{n}^{4}\right]\right]^{m} .
\end{aligned}
$$

Dividing (6) by (3),

$$
\begin{aligned}
\frac{f\left(y_{n}\right)}{f^{\prime}\left(z_{n}\right)} & =\frac{1}{m}\left[\frac{\left(\frac{m-1}{m} e_{n}+\frac{c_{m+1}}{m^{2}(m+1)} e_{n}^{2}-\frac{(2+m) c_{m+1}^{2}-2 m c_{m+2}}{m^{3}(m+1)(m+2)} e_{n}^{3}+\mathcal{O}\left[e_{n}^{4}\right]\right)^{m}}{e_{n}^{m-1}+\frac{c_{m+1}}{m} e_{n}^{m}+\frac{c_{m+2}}{(m+1) m} e_{n}^{m+1}+\mathcal{O}\left[e_{n}^{m+2}\right]}\right] \\
& =\tilde{\mathcal{E}}_{m}^{(1)} e_{n}+\tilde{\mathcal{E}}_{m}^{(2)} e_{n}^{2}+\tilde{\mathcal{E}}_{m}^{(3)} e_{n}^{3}+\mathcal{O}\left[e_{m}^{4}\right],
\end{aligned}
$$

where

$$
\begin{aligned}
& \tilde{\mathcal{E}}_{m}^{(1)}=(m-1)^{m} m^{-(m+1)} ; \\
& \tilde{\mathcal{E}}_{m}^{(2)}=-\frac{(m-1)^{m-1} m^{-(m+2)}\left(m^{2}-m-1\right) c_{m+1}}{1+m} ; \\
& \tilde{\mathcal{E}}_{m}^{(3)}=\frac{(m-1)^{m-1} m^{-(m+3)}\left[(m+2)\left[2 m^{3}-2 m^{2}-5 m-2\right] c_{m+1}^{2}-2 m(m-2)(m+1)^{2} c_{m+2}\right]}{2(m+1)^{2}(m+1)} .
\end{aligned}
$$

Finally, from (5) and (7)

$$
\begin{aligned}
e_{\mathrm{n}+1} & =z_{n+1}-\alpha=y_{n}-\alpha-\gamma \frac{f\left(y_{n}\right)}{f^{\prime}\left(z_{n}\right)} \\
& =\left(\frac{m-1}{m}-\gamma \tilde{\mathcal{E}}_{m}^{(1)}\right) e_{n}+\left(\frac{c_{m+1}}{m^{2}(m+1)}-\gamma \tilde{\mathcal{E}}_{m}^{(2)}\right) e_{n}^{2}+\left(\frac{2 m c_{m+2}-(2+m) c_{m+1}^{2}}{m^{3}(m+1)(m+2)}-\gamma \tilde{\mathcal{E}}_{m}^{(3)}\right) e_{n}^{3}+\mathcal{O}\left[e_{n}^{4}\right] \\
& =\mathcal{E}_{m}^{(1)}(\gamma) e_{n}+\mathcal{E}_{m}^{(2)}(\gamma) e_{n}^{2}+\mathcal{E}_{m}^{(3)}(\gamma) e_{n}^{3}+\mathcal{O}\left[e_{n}^{4}\right],
\end{aligned}
$$

and the proof is finished.

From the previous result, it has been proved that the order of convergence depends both on the parameter determining the method $(\gamma)$ and the multiplicity of the root $(m)$. Moreover, unlike the case involving simple roots, where the order of convergence of the Traub's family is at least two [4], by considering roots with multiplicity $m>1$, the minimum order attainable is now one. However, as can be seen in Figure 1, the values of $\gamma$ giving rise to quadratic convergence, are located at $\gamma_{m}=m^{m}(m-1)^{1-m}$, that is, quadratic convergence can be always obtained for a value of parameter $\gamma$ depending on the multiplicity of the root.

In the particular case of polynomial $p(z)=(z-a)^{2}(z-b)$, the error equation is given by:

$$
e_{n+1}=\left(\frac{4-\gamma}{8}\right) e_{n}+\left(\frac{(4+\gamma) c_{3}}{48}\right) e_{n}^{2}+\left(\frac{6 c_{4}+(\gamma-6) c_{3}^{2}}{144}\right) e_{n}^{3}+\mathcal{O}\left[e_{n}^{4}\right]
$$

Let us note that both Traub $(\gamma=1)$ and Newton $(\gamma=0)$ methods have first order of convergence. Only the case where $\gamma=4$ gives rise to a higher convergence (quadratic), and hence, deserves a special attention in the subsequent analysis.

In the following sections, we deal with the dynamical analysis of the family of methods given by (1), emphasizing the stability and reliability features. In order to find the regions of the parameter space (i.e., the members of the family) where the behavior is suitable in terms of numerical stability, we compute the fixed and the critical points of the corresponding rational function. 


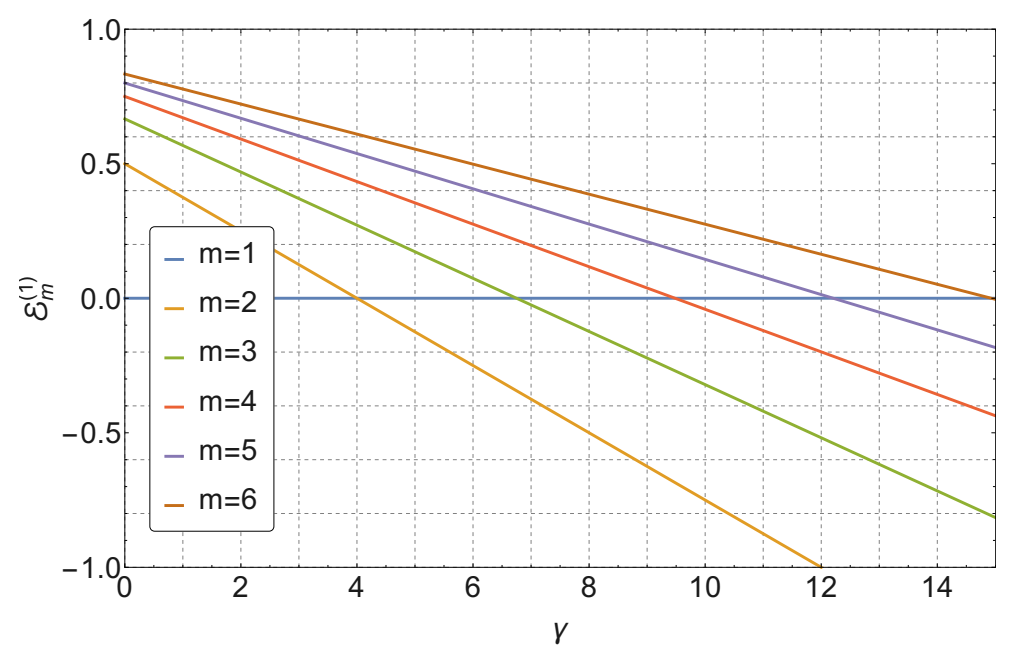

Figure 1: First coefficient on the error equation as a function of $\gamma$ for different values of the multiplicity

\section{Basic concepts}

By using the tools of complex dynamics, we will study the general convergence of family (1) on cubic polynomials with multiple roots. Taking into account the Scaling Theorem, that is satisfied in this case (see [5]), the roots of a polynomial can be transformed by an affine map with no qualitative changes on the dynamical behavior of the family. Thus, by using the cubic polynomial $p(z)=(z-a)^{2}(z-b)$, the operator of the family is given by the rational function

$$
T_{p, \gamma, a, b}(z)=z+\frac{(a-z)(z-b)\left[2(a+b-2 z)^{2}(z-b) \gamma-(a+2 b-3 z)^{3}\right]}{(a+2 b-3 z)^{4}}
$$

depending on the parameters $\gamma, a$ and $b$.

Blanchard, in [17], by considering the conjugacy map

$$
h(z)=\frac{z-a}{z-b}
$$

with the following properties:

$$
\text { (i) } h(\infty)=1, \quad \text { (ii) } h(a)=0, \quad \text { (iii) } h(b)=\infty,
$$

proved that, for quadratic polynomials, Newton's operator is conjugate to the rational map $z^{2}$. Similarly, the operator $T_{p, \gamma, a, b}(z)$ on cubic polynomials is conjugated to the operator $Q_{\gamma}(z)$ :

$$
Q_{\gamma}(z)=\left[h \circ T_{p, \gamma, a, b} \circ h^{-1}\right](z)=\frac{z(z+1)\left[2(z+1) \gamma-(z+2)^{3}\right]}{2 z(z+1)^{2} \gamma-2(z+2)^{3}} .
$$

We observe that the parameters $a$ and $b$ have been obviated in $Q_{\gamma}(z)$.

Now, we are going to recall some dynamical concepts of complex dynamics (see [18]) that will be used in this work. Given a rational function $R: \hat{\mathbb{C}} \rightarrow \hat{\mathbb{C}}$, where $\hat{\mathbb{C}}$ is the Riemann sphere, the orbit of a point $z_{0} \in \hat{\mathbb{C}}$ is defined as the set of successive images of $z_{0}$ by the rational function:

$$
\left\{z_{0}, R\left(z_{0}\right), R^{2}\left(z_{0}\right), \ldots, R^{n}\left(z_{0}\right), \ldots\right\}
$$

We analyze the phase plane of the map $R$ by classifying the starting points from the asymptotic behavior of their orbits. A $z_{0} \in \hat{\mathbb{C}}$ is called a fixed point if $R\left(z_{0}\right)=z_{0}$. A periodic point $z_{0}$ of period $p>1$ is a point such that $R^{p}\left(z_{0}\right)=z_{0}$ and $R^{k}\left(z_{0}\right) \neq z_{0}$, for $k<p$. A pre-periodic point is a point $z_{0}$ that is not periodic but there exists a $k>0$ such that $R^{k}\left(z_{0}\right)$ is periodic. A critical point $z_{0}$ is a point where the derivative of the rational function vanishes, $R^{\prime}\left(z_{0}\right)=0$. Moreover, a fixed point $z_{0}$ is called attractor if $\left|R^{\prime}\left(z_{0}\right)\right|<1$, superattractor if $\left|R^{\prime}\left(z_{0}\right)\right|=0$, repulsor if $\left|R^{\prime}\left(z_{0}\right)\right|>1$ and parabolic if $\left|R^{\prime}\left(z_{0}\right)\right|=1$. 
The basin of attraction of an attractor $\alpha$ is defined as the set of preimages of any order:

$$
\mathcal{A}(\alpha)=\left\{z_{0} \in \hat{\mathbb{C}}: R^{n}\left(z_{0}\right) \rightarrow \alpha, n \rightarrow \infty\right\} .
$$

The Fatou set of the rational function $R, \mathcal{F}(R)$, is the set of points $z \in \hat{\mathbb{C}}$ whose orbits tend to an attractor (fixed point, periodic orbit or infinity). Its complement in $\hat{\mathbb{C}}$ is the Julia set, $\mathcal{J}(R)$. That means that the basin of attraction of any fixed point belongs to the Fatou set and the boundaries of these basins of attraction belong to the Julia set.

\section{Analysis of the fixed and critical points}

Points $z=0, z=\infty$ are fixed points of operator $Q_{\gamma}(z)$, that is, are roots of the equation $Q_{\gamma}(z)=z$ (indeed, $z=\infty$ is a fixed point associated to the operator $Q_{\gamma}(z)$ if and only if $\lim _{z \rightarrow 0} \frac{1}{Q_{\gamma}\left(\frac{1}{z}\right)}=0$ ), as well as a set of strange fixed points. However, due to the non trivial form of the operator (11), in order to calculate the strange fixed points, it becomes necessary to analyze separately the behavior of its numerator and denominator. The roots of the denominator, i.e., the poles of the operator $Q_{\gamma}(z)$, are given by

$$
\begin{aligned}
P_{Q_{\gamma}}^{(1)}(\gamma) & =\frac{1}{3(\gamma-1)}\left[6-2 \gamma+\frac{\gamma(\gamma+15)}{\rho(\gamma)}+\rho(\gamma)\right], \\
P_{Q_{\gamma}}^{(2)}(\gamma) & =\frac{1}{24(\gamma-1)}\left[16(3-\gamma)+\frac{4 \gamma(\gamma+15)(-1-i \sqrt{3})}{\rho(\gamma)}-4(1-i \sqrt{3}) \rho(\gamma)\right], \\
P_{Q_{\gamma}}^{(3)}(\gamma) & =\frac{1}{24(\gamma-1)}\left[16(3-\gamma)+\frac{4 \gamma(\gamma+15)(-1+i \sqrt{3})}{\rho(\gamma)}-4(1+i \sqrt{3}) \rho(\gamma)\right],
\end{aligned}
$$

where

$$
\rho(\gamma)=\left[3 \sqrt{3} \sqrt{\gamma^{2}(\gamma-1)^{2}(\gamma+27)}+\gamma(27+\gamma(\gamma+36))\right]^{1 / 3}
$$
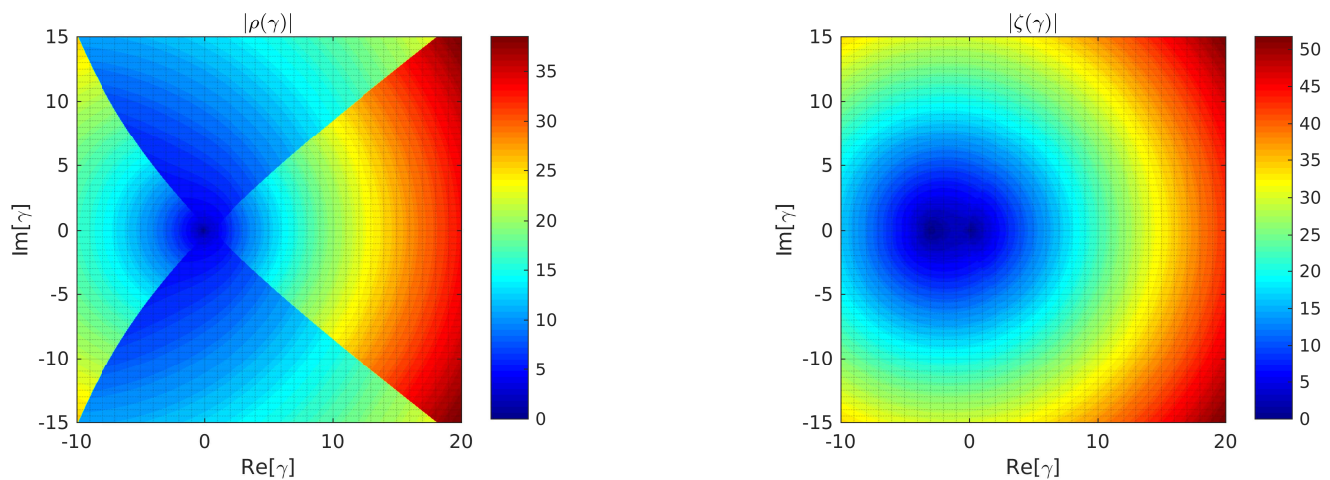

Figure 2: Parameter space showing $|\rho(\gamma)|$ and $|\zeta(\gamma)|$ as a function of the real and imaginary parts of $\gamma$

From this last expressions, we can directly observe that $\gamma=1$ gives rise to a singularity. Additionally, it can be showed that $\rho(\gamma)=0$ if and only if $\gamma=0$ (see Figure 2). Therefore, since $\gamma=0$ (Newton's method) and $\gamma=1$ (Traub's scheme) are special cases (yielding indeterminate forms and singularities, respectively), also deserve further analysis. Let us remark that for each value of $\gamma$, there are three different complex values of $\rho(\gamma)$, which we will denote as $\left\{\rho_{1}(\gamma), \rho_{2}(\gamma), \rho_{3}(\gamma)\right\}$. If we assume that $\rho_{1}(\gamma) \equiv \rho(\gamma)$ is the so called principal cubic root (usually defined as the cubic root with the largest real part), the other ones can be written as $\rho_{m}(\gamma)=\rho(\gamma) e^{i \frac{2 \pi}{3}(m-1)}, m=2,3$. It can be proved that

$$
\begin{aligned}
& \left.P_{Q_{\gamma}}^{(1)}(\gamma)\right|_{\rho_{1}(\gamma)}=\left.P_{Q_{\gamma}}^{(2)}(\gamma)\right|_{\rho_{3}(\gamma)}=\left.P_{Q_{\gamma}}^{(3)}(\gamma)\right|_{\rho_{2}(\gamma)}, \\
& \left.P_{Q_{\gamma}}^{(1)}(\gamma)\right|_{\rho_{2}(\gamma)}=\left.P_{Q_{\gamma}}^{(2)}(\gamma)\right|_{\rho_{1}(\gamma)}=\left.P_{Q_{\gamma}}^{(3)}(\gamma)\right|_{\rho_{3}(\gamma)}, \\
& \left.P_{Q_{\gamma}}^{(1)}(\gamma)\right|_{\rho_{3}(\gamma)}=\left.P_{Q_{\gamma}}^{(2)}(\gamma)\right|_{\rho_{2}(\gamma)}=\left.P_{Q_{\gamma}}^{(3)}(\gamma)\right|_{\rho_{1}(\gamma)}
\end{aligned}
$$


On the other hand, disregarding these values for the $\gamma$ parameter, the strange fixed points are given by the following expressions:

$$
\begin{aligned}
e x_{1}(\gamma) & =1, \text { for } \gamma \neq 27 / 4, \\
e x_{2}(\gamma) & =\frac{1}{3}\left[\zeta(\gamma)+\frac{4 \gamma(\gamma+3)}{\zeta(\gamma)}-2(\gamma+3)\right], \\
e x_{3}(\gamma) & =-\frac{1}{6}\left[\zeta(\gamma)(1-i \sqrt{3})+\frac{4 \gamma(\gamma+3)(1+i \sqrt{3})}{\zeta(\gamma)}+4(\gamma+3)\right], \\
e x_{4}(\gamma) & =-\frac{1}{6}\left[\zeta(\gamma)(1+i \sqrt{3})+\frac{4 \gamma(\gamma+3)(1-i \sqrt{3})}{\zeta(\gamma)}+4(\gamma+3)\right],
\end{aligned}
$$

where

$$
\zeta(\gamma)=\left[3 \sqrt{3} \sqrt{\gamma^{2}(27+8 \gamma)}-\gamma(27+\gamma(36+8 \gamma))\right]^{1 / 3}
$$

Note that $\zeta(\gamma)=0$ if and only if $\gamma=0$ or $\gamma=-3$ (see Figure 2). As above, taking into account the cubic roots, for the strange fixed points it can also be proved that

$$
\begin{aligned}
\left.e x_{2}(\gamma)\right|_{\zeta_{1}(\gamma)} & =\left.e x_{3}(\gamma)\right|_{\zeta_{3}(\gamma)}=\left.e x_{4}(\gamma)\right|_{\zeta_{2}(\gamma)}, \\
\left.e x_{2}(\gamma)\right|_{\zeta_{2}(\gamma)} & =\left.e x_{3}(\gamma)\right|_{\zeta_{1}(\gamma)}=\left.e x_{4}(\gamma)\right|_{\zeta_{3}(\gamma)}, \\
\left.e x_{2}(\gamma)\right|_{\zeta_{3}(\gamma)} & =\left.e x_{3}(\gamma)\right|_{\zeta_{2}(\gamma)}=\left.e x_{4}(\gamma)\right|_{\zeta_{1}(\gamma)}
\end{aligned}
$$

Taking into account the previous results, the main relationship between the strange fixed points are summarized by means of the following result. Let us remark that, although case $\gamma=0$ corresponds to Newton's method, the associate operator is not $z^{2}$, as we are working on a polynomial with multiple roots.

Lemma 1. Let $Q_{\gamma}(z)$ be the rational function resulting from applying family (1) on polynomial $p(z)=(z-a)^{2}(z-b)$, conjugated by means of Möbius map. Then, the number of simple strange fixed points of operator $Q_{\gamma}(z)$ is four (denoted by $\left.\operatorname{ex}_{i}(\gamma), i=1,2,3,4\right)$, except in the following cases:

i) If $\gamma=0$, the operator's expression is $Q_{0}(z)=z(z+1) / 2$, and the only simple strange fixed point is ex $x_{1}(\gamma)=1$.

ii) If $\gamma=27 / 4$, there are three simple strange fixed points, ex $x_{2}(27 / 4)$, ex $x_{3}(27 / 4)$ and ex $x_{4}(27 / 4)$, being associated with the rational function

$$
Q_{27 / 4}(z)=z(1+z)\left[\frac{27(z+1)-2(z+2)^{3}}{27 z(z+1)^{2}-4(z+2)^{3}}\right] .
$$

iii) If $\gamma=-27 / 8$, then $\zeta(-27 / 8)=-9 / 4$ and e $x_{1}(-27 / 8)=e x_{3}(-27 / 8)=e x_{4}(-27 / 8)=1$. So, there exists only two strange fixed points, one of them with multiplicity three. The operator associated to this parameter is given by:

$$
Q_{-27 / 8}(z)=z(z+1)\left[\frac{27(z+1)+4(z+2)^{3}}{27 z(z+1)^{2}+8(z+2)^{3}}\right] .
$$

iv) If $\gamma=-4$, then $\zeta(-4)=\sqrt[3]{44+12 i \sqrt{15}}$, and ex $(-4)=0$. Hence, there are only three simple strange fixed points, being the associated operator

$$
Q_{-4}(z)=z(z+1)\left[\frac{8(z+1)+(z+2)^{3}}{8 z(z+1)^{2}+2(z+2)^{3}}\right] .
$$

In order to determine the critical points, we calculate the first derivative of $Q_{\gamma}(z)$,

$$
Q_{\gamma}^{\prime}(z)=\frac{(z+2)^{3}(2 z+1)}{2}\left[\frac{(z+2)^{3}-\gamma\left(z^{3}+z+2\right)}{\left((z+2)^{3}-\gamma z(z+1)^{2}\right)^{2}}\right]
$$

The relevance of the knowledge of the critical points relies on a classical result which establishes that each invariant Fatou component is associated with, at least, one critical point. In the case of quadratic polynomials with simple roots [4], where the order of convergence is at least two, it is clear that the image by the conjugacy map (10) of the roots of the polynomial 
are critical points, giving rise to their respective Fatou components. However, in this case, the order of convergence is one except for $\gamma=4$, that is two. Therefore, in the analysis of the critical points we must study separately the cases $\gamma=4$ and $\gamma \neq 4$.

As we have stated in the previous section dealing with the fixed points, we firstly analyze the poles of the rational function $Q_{\gamma}^{\prime}(z)$, which are given by:

$$
\begin{aligned}
P_{Q_{\gamma}^{\prime}}^{(1)}(\gamma) & =\frac{1}{3(1-\gamma)}\left[2(\gamma-3)+\frac{\gamma(\gamma+15)}{\tilde{\rho}(\gamma)}+\tilde{\rho}(\gamma)\right], \\
P_{Q_{\gamma}^{\prime}}^{(2)}(\gamma) & =\frac{1}{6(1-\gamma)}\left[4(\gamma-3)+\frac{\gamma(\gamma+15)(-1-i \sqrt{3})}{\tilde{\rho}(\gamma)}-(1-i \sqrt{3}) \tilde{\rho}(\gamma)\right], \\
P_{Q_{\gamma}^{\prime}}^{(3)}(\gamma) & =\frac{1}{6(1-\gamma)}\left[4(\gamma-3)+\frac{\gamma(\gamma+15)(-1+i \sqrt{3})}{\tilde{\rho}(\gamma)}-(1+i \sqrt{3}) \tilde{\rho}(\gamma)\right],
\end{aligned}
$$

where

$$
\tilde{\rho}(\gamma)=\left[3 \sqrt{3} \sqrt{\gamma^{2}(\gamma-1)^{2}(\gamma+27)}-\gamma(27+\gamma(\gamma+36))\right]^{1 / 3}
$$
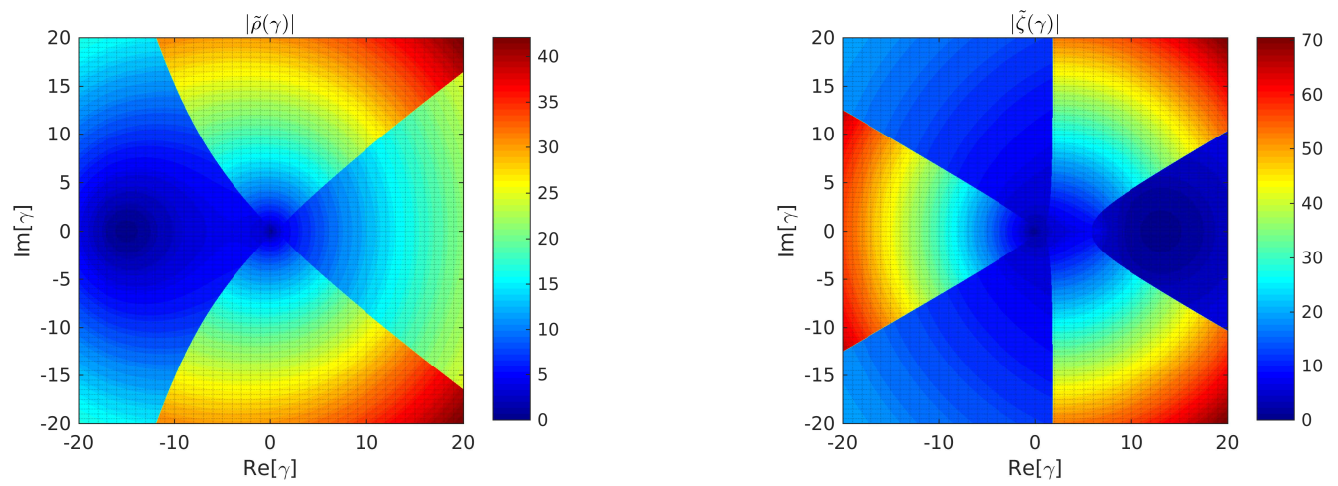

Figure 3: Parameter space showing $|\tilde{\rho}(\gamma)|$ and $|\tilde{\zeta}(\gamma)|$ as a function of the real and imaginary parts of $\gamma$

We observe that $\gamma=1$ gives rise to a singularity, and it can be showed that $\tilde{\rho}(\gamma)=0$ if and only if $\gamma=0$ or $\gamma=-15$ (see Figure 3). Again, taking into account the cubic roots, for the poles of the first derivative of $Q_{\gamma}(z)$ it can be proved that

$$
\begin{gathered}
P_{\left.Q_{\gamma}^{\prime}(\gamma)\right|_{\tilde{\rho}_{1}(\gamma)} ^{(1)}}=P_{\left.Q_{\gamma}^{\prime}(\gamma)\right|_{\tilde{\rho}_{3}(\gamma)} ^{(2)}}=P_{\left.Q_{\gamma}^{\prime}(\gamma)\right|_{\tilde{\rho}_{2}(\gamma)} ^{(3)},}, \\
P_{\left.Q_{\gamma}^{\prime}(\gamma)\right|_{\tilde{\rho}_{2}(\gamma)}}^{(1)}=P_{\left.Q_{\gamma}^{\prime}(\gamma)\right|_{\tilde{\rho}_{1}(\gamma)} ^{(2)}}=\left.P_{Q_{\gamma}^{\prime}}^{(3)}(\gamma)\right|_{\tilde{\rho}_{3}(\gamma)}, \\
P_{\left.Q_{\gamma}^{\prime}(\gamma)\right|_{\tilde{\rho}_{3}(\gamma)} ^{(1)}}=P_{\left.Q_{\gamma}^{\prime}(\gamma)\right|_{\tilde{\rho}_{2}(\gamma)} ^{(2)}}=\left.P_{Q_{\gamma}^{\prime}}^{(3)}(\gamma)\right|_{\tilde{\rho}_{1}(\gamma)} .
\end{gathered}
$$

By considering this results, we establish the following lemma:

Lemma 2. By analyzing the equation $Q_{\gamma}^{\prime}(z)=0$, we obtain:

i) If $\gamma \neq 0$, there are two free critical points: $c r_{1}(\gamma)=-1 / 2$ and $c r_{2}(\gamma)=-2$, where the multiplicity of cr $r_{2}(\gamma)$ is always 3. Let us note that, since $c r_{1}(\gamma)=\left[c r_{2}(\gamma)\right]^{-1}$, they are not independent. On the other hand, if $\gamma=0$, there exists only one simple free critical point: $c r_{1}(\gamma)=-1 / 2$.

ii) Only in case $\gamma=4, z=0$ and $z=\infty$ (related to the roots of the polynomial by means of Möbius map) are critical points giving rise to their respective Fatou components. Additionally, there exist more free critical points: $\mathrm{cr}_{1}(\gamma)$, $c r_{2}(\gamma)$ and

$$
\begin{aligned}
c r_{3}(4) & =\frac{1}{3}[3+\sqrt{33}] \\
c r_{4}(4) & =\frac{1}{3}[3-\sqrt{33}] .
\end{aligned}
$$


iii) If $\gamma=-27$, there are four free critical points: $c r_{2}(\gamma)$ with multiplicity 3 and

$$
\begin{aligned}
& c r_{3}(-27)=\frac{1}{14}\left[-1-6 \sqrt[3]{25}\left(\frac{2}{-43+7 \sqrt{41}}\right)^{1 / 3}+3\left(\frac{5}{2}(-43+7 \sqrt{41})\right)^{1 / 3}\right], \\
& c r_{4}(-27)=-\frac{1}{14}+\frac{3 \sqrt[3]{25}(1+i \sqrt{3})}{7 \sqrt[3]{4}(-43+7 \sqrt{41})^{1 / 3}}-\frac{3}{28}(1-i \sqrt{3})\left[\frac{5}{2}(-43+7 \sqrt{41})\right]^{1 / 3}, \\
& c r_{5}(-27)=-\frac{1}{14}+\frac{3 \sqrt[3]{25}(1-i \sqrt{3})}{7 \sqrt[3]{4}(-43+7 \sqrt{41})^{1 / 3}}-\frac{3}{28}(1+i \sqrt{3})\left[\frac{5}{2}(-43+7 \sqrt{41})\right]^{1 / 3} .
\end{aligned}
$$

Let us notice that $c r_{4}(-27)=\left[c r_{5}(-27)\right]^{*}$, where the asterisk denotes complex conjugation.

iv) If $\gamma=27 / 4$, we can find three free critical points, $\operatorname{cr}_{1}(\gamma)=-1 / 2, c r_{2}(\gamma)=-2$ and $c r_{4}(27 / 4)=-22 / 23$.

v) If $\gamma=16 / 7$, there exists four free critical points, $c r_{1}(\gamma)=-1 / 2, c r_{2}(\gamma)=-2, c r_{3}(16 / 7)=6$ and $_{c r}(16 / 7)=$ $-2 / 3$, being the multiplicity of $\mathrm{cr}_{4}(16 / 7)$, equal to 2 .

vi) If $\gamma=27 / 11$ there are four free critical points, $c r_{1}(\gamma)=-1 / 2, c r_{2}(\gamma)=-2$,

$$
\begin{aligned}
& c r_{3}(27 / 11)=\frac{1}{16}(37+3 \sqrt{273}), \\
& c r_{4}(27 / 11)=\frac{1}{16}(37-3 \sqrt{273}) .
\end{aligned}
$$

In this case, the multiplicity of $\operatorname{cr}_{1}(\gamma)=-1 / 2$ is 2 .

vii) If $\gamma=1$, there exist six free critical points, $c r_{1}(\gamma), c r_{2}(\gamma), c r_{3}(1)=\frac{1}{12}[-11-i \sqrt{23}]$ and $c r_{4}(1)=\left[c r_{3}(1)\right]^{*}$.

viii) In any other case the free critical points are $\operatorname{cr}_{1}(\gamma)=-1 / 2, \operatorname{cr}_{2}(\gamma)=-2$ and the following:

$$
\begin{aligned}
c r_{3}(\gamma) & =\frac{1}{9(\gamma-1)}\left[18-\frac{3 \sqrt[3]{9} \gamma(\gamma-13)}{\tilde{\zeta}(\gamma)}+3 \sqrt[3]{3} \tilde{\zeta}(\gamma)\right], \\
c r_{4}(\gamma) & =\frac{1}{18(\gamma-1)}\left[36+\frac{3 \sqrt[3]{9} \gamma(\gamma-13)(1+i \sqrt{3})}{\tilde{\zeta}(\gamma)}+3 \sqrt[3]{3}(-1+i \sqrt{3}) \tilde{\zeta}(\gamma)\right], \\
c r_{5}(\gamma) & =\frac{1}{18(\gamma-1)}\left[36+\frac{3 \sqrt[3]{9} \gamma(\gamma-13)(1-i \sqrt{3})}{\tilde{\zeta}(\gamma)}+3 \sqrt[3]{3}(-1-i \sqrt{3}) \tilde{\zeta}(\gamma)\right],
\end{aligned}
$$

where

$$
\tilde{\zeta}(\gamma)=\left[\sqrt{3} \sqrt{\gamma^{2}(\gamma-1)^{2}(4 \gamma-27)(7 \gamma-16)}-9 \gamma(-4+\gamma(\gamma-5))\right]^{1 / 3} .
$$

In the last expressions we can observe that, once again $\gamma=1$ gives rise to a singularity, and $\tilde{\zeta}(\gamma)=0$ if and only if $\gamma=0$ or $\gamma=13$ (see Figure 3). Moreover, for the free critical points it can be proved that

$$
\begin{aligned}
& \left.c r_{3}(\gamma)\right|_{\tilde{\zeta}_{1}(\gamma)}=\left.c r_{4}(\gamma)\right|_{\tilde{\zeta}_{3}(\gamma)}=\left.c r_{5}(\gamma)\right|_{\tilde{\zeta}_{2}(\gamma)}, \\
& \left.c r_{3}(\gamma)\right|_{\tilde{\zeta}_{2}(\gamma)}=\left.c r_{4}(\gamma)\right|_{\tilde{\zeta}_{1}(\gamma)}=\left.c r_{5}(\gamma)\right|_{\tilde{\zeta}_{3}(\gamma)}, \\
& \left.c r_{3}(\gamma)\right|_{\tilde{\zeta}_{3}(\gamma)}=\left.c r_{4}(\gamma)\right|_{\tilde{\zeta}_{2}(\gamma)}=\left.c r_{5}(\gamma)\right|_{\tilde{\zeta}_{1}(\gamma)} .
\end{aligned}
$$

Some of these properties determine the complexity of the operator. From the previous results, let us remark that:

- Since $c r_{1}(\gamma)=\left[c r_{2}(\gamma)\right]^{-1}$, at most, there are four independent free critical points. However, there exists regions of the parameter space where appear more relations between the other free critical points. In particular, it can be proved that $c r_{4}(\gamma)=\left[c r_{5}(\gamma)\right]^{*}$ on the complement of the real interval $\gamma \in[16 / 7,13]$. In Figure 4, the real values of the $\gamma$ parameter are highlighted if the strange fixed points $\left(e x_{3}(\gamma)\right.$ and $\left.e x_{4}(\gamma)\right)$ and the free critical points $\left(\operatorname{cr}_{4}(\gamma)\right.$ and $\left.\operatorname{cr}_{5}(\gamma)\right)$ are related by means of the complex conjugacy.

- The free critical point $c r_{2}(\gamma)=-2$ is a pre-image of $z=1$, i.e., $Q_{\gamma}(-2)=1$ for all $\gamma \in \mathbb{C}$.

- Whenever $\gamma=4$, the order of convergence of the method is quadratic, and then the roots $z=0$ and $z=\infty$ are critical points giving rise to their respective Fatou components. In that case, there are three independent free critical points.

- If $\gamma=0$, there exists only one strange fixed point, $e x_{1}(\gamma)=1$ and one free critical point $c r_{1}(\gamma)=-1 / 2$. 


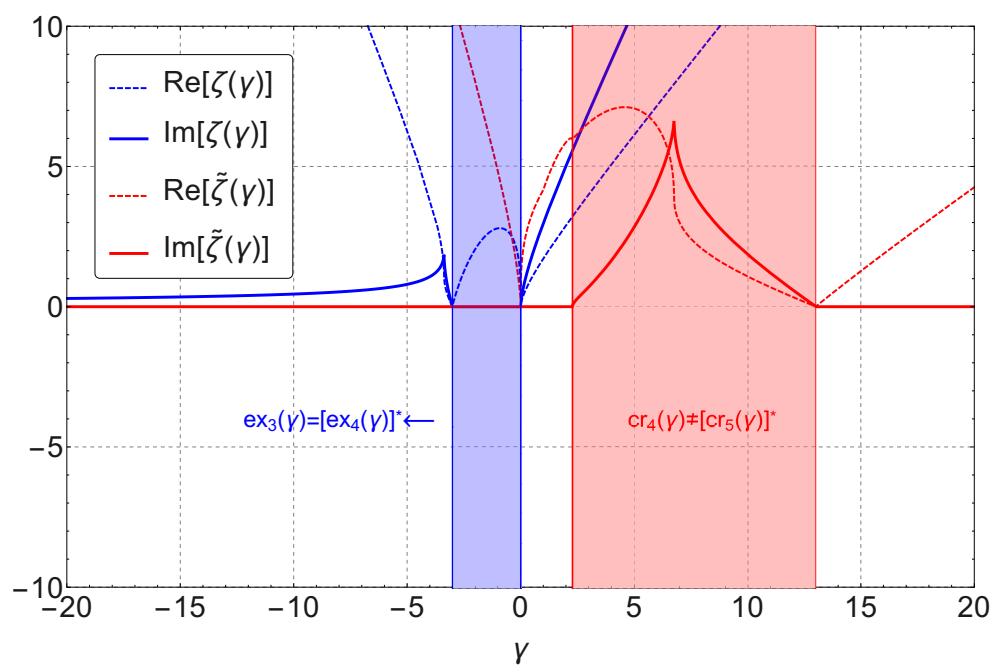

Figure 4: Real and imaginary parts of the functions $\zeta(\gamma)$ and $\tilde{\zeta}(\gamma)$

\section{Stability of the fixed points}

As we will see below, not only the number but also the stability of the fixed points depend on the parameter of the family. The relevance of this study yields in the fact that the existence of attracting strange fixed points can make the iterative scheme converge to a "false" solution.

When the order of convergence of the family is at least two, it is clear that the origin and $\infty$ are always superattractive fixed points. However, the stability of the other fixed point changes depending on the values of the parameter $\gamma$, and gives us interesting numerical information. In the following results we establish the stability of the fixed points.

As family (1) has second order of convergence for simple roots, the fixed point $z=\infty$ is superattracting for all values of $\gamma$. However, the stability of $z=0$ is determined in the following result, where a region of the complex plane is defined being simultaneously basin of attraction of both roots of the polynomial.

Theorem 2. The analysis of the stability of the fixed point $z=0$ shows that:

i) If $|4-\gamma|<8$, then $z=0$ is an attractor and it is a superattractor if $\gamma=4$.

ii) If $|4-\gamma|=8$, then $z=0$ is a parabolic point.

iii) If $|4-\gamma|>8$, then $z=0$ is a repulsor.

Proof. It is easy to prove that $Q_{\gamma}^{\prime}(0)=\frac{1}{16}[8-2 \gamma]$ and. So,

$$
\left|\frac{8-2 \gamma}{16}\right| \leq 1 \text { is equivalent to } 0 \leq|4-\gamma| \leq 8
$$

Analyzing the equation $Q_{\gamma}^{\prime}(0)=0$, it is straightforward to observe that the unique value of $\gamma$ verifying $Q_{\gamma}^{\prime}(0)=0$ is $\gamma=4$. Therefore, the fixed points $z=0$ and $z=\infty$ are superattractors if and only if the order of convergence of the family is two. Moreover, if $\gamma$ satisfies $0<|4-\gamma|<8$, the fixed points are attractors. If $|4-\gamma|=8$, then $\left|Q_{\gamma}^{\prime}(0)\right|=0$ and the fixed points are parabolic points. And finally, if $|4-\gamma|>8$ then the fixed points are repulsors.

Let us remark that the disk $|4-\gamma|<8$, where the fixed points $z=0$ and $\infty$ are attractors, coincides with the region with good numerical behavior when the family of methods is applied to quadratic polynomials with simple roots (see [4]). Similar results can be proved for the rest of strange fixed points.

Theorem 3. The character of the strange fixed point ex $x_{1}(\gamma)=1, \gamma \neq 27 / 4$, is:

i) If $\left|\frac{27}{4}-\gamma\right|>\frac{81}{8}$, then $e x_{1}(\gamma)=1$ is an attractor, but it cannot be a superattractor.

ii) If $\left|\frac{27}{4}-\gamma\right|=\frac{81}{8}$, then $e x_{1}(\gamma)=1$ is a parabolic point.

iii If $\left|\frac{27}{4}-\gamma\right|<\frac{81}{8}$, then $\operatorname{ex}_{1}(\gamma)=1$ is a repulsor. 

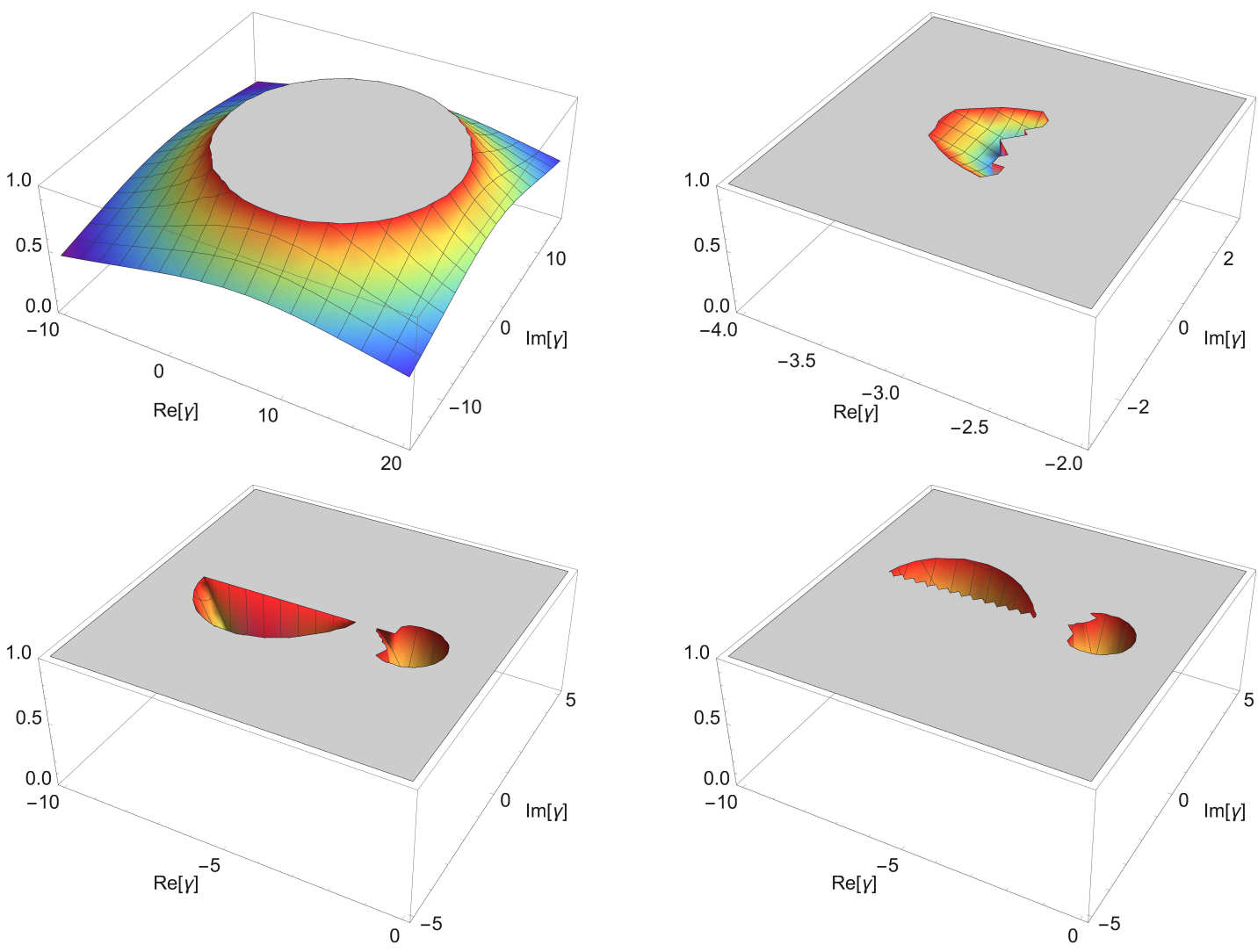

Figure 5: Stability regions of $e x_{i}(\gamma), i=1,2,3,4$

The proof of this theorem is analogous to that of Theorem 2, by using the stability function of $e x_{1}(\gamma)=1$,

$$
Q_{\gamma}^{\prime}(1)=\frac{81}{2(27-4 \gamma)} .
$$

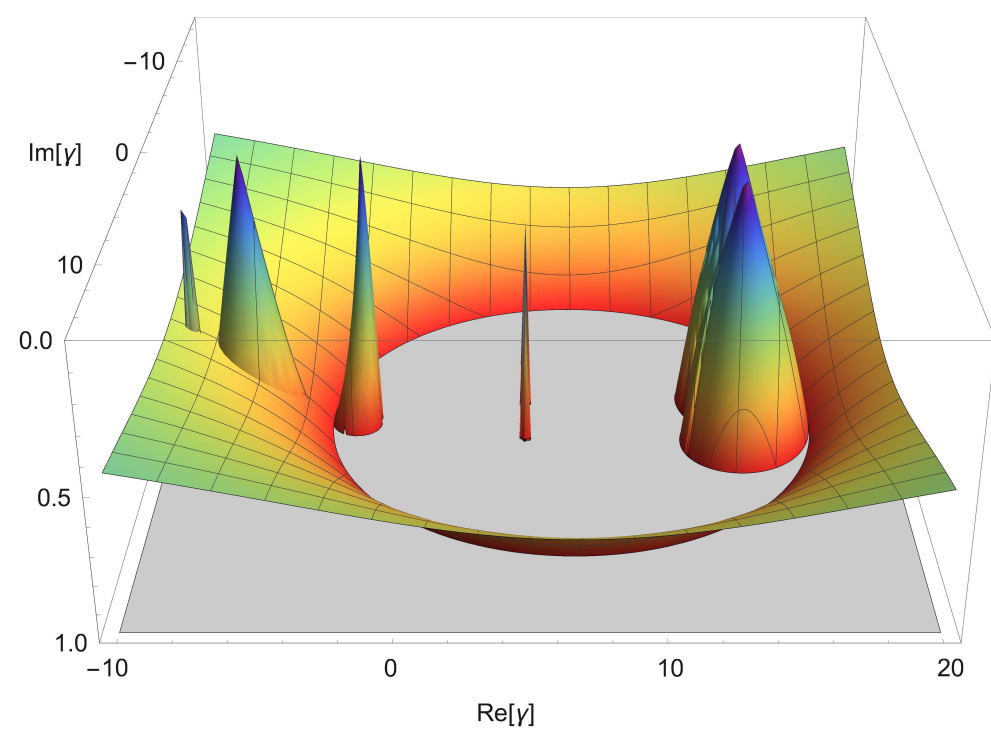

Figure 6: Stability regions of strange fixed points

The analytical expressions that determine the character of the rest of strange fixed points depending on $\gamma$ are very 
complicated. Instead, we analyze the stability by means of graphical representations. In Figure 5, we represent the stability region associated to each strange fixed points previously calculated, i.e., we plot $\left|Q_{\gamma}^{\prime}\left(e x_{i}(\gamma)\right)\right|, i=1,2,3,4$, as a function of the real and imaginary components of the $\gamma$ parameter. It can be checked that $\operatorname{ex}_{3}(\gamma)$ is superattracting if $\gamma=-1.82812-0.181546 i$ and there exist two values of $\gamma$ where $e x_{4}(\gamma)$ is superattracting, $\gamma=-6.75$ and $\gamma=$ $-1.82812+0.181546 i$. In Figure 6 we show all stability regions, including those corresponding to those of the set of 2-periodic points, obtained numerically as $\gamma$-dependent roots of a 20th-degree polynomial. Nevertheless, it can be numerically checked that these periodic orbits can be superattracting if $\gamma=13.081$ or $\gamma=13.5406 \pm 1.8965 i$.

\section{The parameter space}

From the previous analysis, it is clear that the dynamical behavior of the rational operator $Q_{\gamma}(z)$ associated with each value of the $\gamma$ parameter can be very different. Several parameter spaces associated with each free critical point of the Traub's-type family of iterative methods are obtained by associating each point of the parameter plane with a complex value of $\gamma$, i.e., with an element of the family (1). Every value of $\gamma$ belonging to the same connected component of the parameter space gives rise to subsets of schemes of family (1) with similar dynamical behavior. So, it is interesting to find regions of the parameter plane as much stable as possible, because these values of $\gamma$ will give us the best member of the family in terms of numerical stability. As $c r_{1}(\gamma)=\left[c r_{2}(\gamma)\right]^{-1}$ we have at most four independent free critical points (see
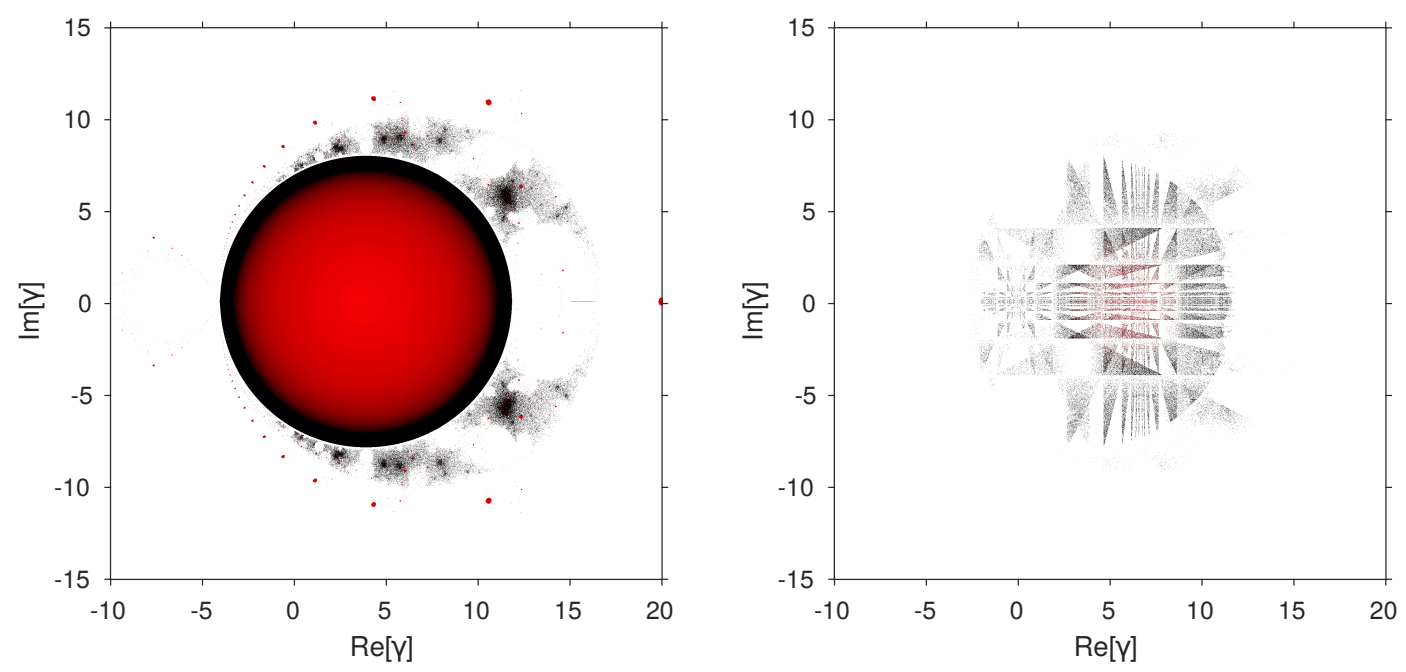

Figure 7: Parameter spaces $P_{1}$ (left) and $P_{2}$ (right) associated to $c r_{1}(\gamma)$ and $c r_{2}(\gamma)$, resp.
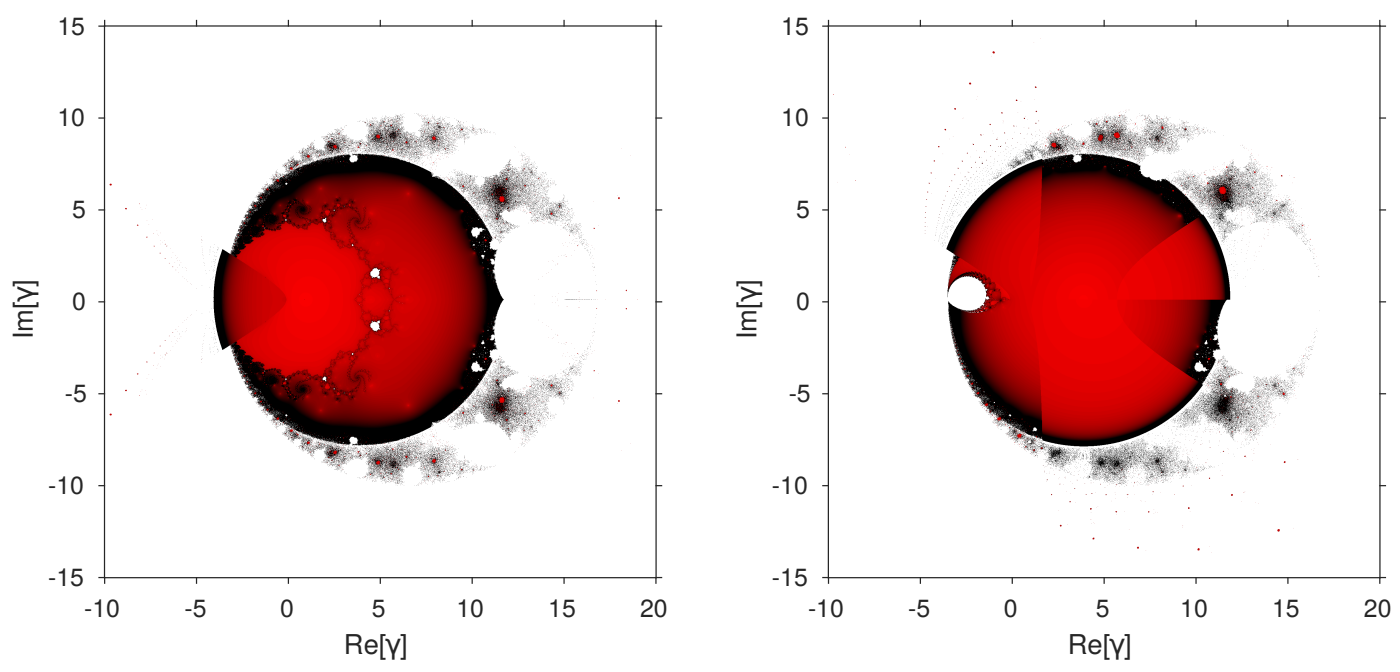

Figure 8: Parameter spaces $P_{3}$ (left) and $P_{4}$ (right) associated to $c r_{3}(\gamma)$ and $c r_{4}(\gamma)$, resp. 
Lemma 2), so we can obtain different parameter planes, with complementary information. When we consider the free critical point $\mathrm{cr}_{1}(\gamma)=-1 / 2$ as a starting point of the iterative scheme of the family associated to each complex value of $\gamma$, we paint this point of the complex plane in red if the method converges to any of the roots (zero and infinity) and they are white in other cases. The color used is brighter when the number of iterations is lower. Then, the parameter plane $P_{1}$ is obtained (see Figure 7). Each parameter plane has been generated by using the routines described in [19], for values of $\gamma$ in $[-10,20] \times[-15,15]$, with a mesh of $2000 \times 2000$ points. The maximum number of iterations involved has been 500 , and $10^{-3}$ the tolerance used as a stopping criterium.

A similar procedure can be carried out with the free critical points $\operatorname{cr}_{i}(\gamma), i=2,3,4,5$, obtaining in each case the corresponding parameter plane $P_{i}$ (see Figures 7 and 8). Let us remark that all parameter planes have common regions: the disk $D_{1}$ that defines the values of $\gamma$ where $e x_{1}(\gamma)=1$ is attractive (defined in Theorem 3) and the red disk $D_{2}$ where $z=0$ is attracting, defined in Theorem 2. In the case of $P_{2}$ it can be proved that $Q_{\gamma}(-2)=1$, and 1 is a repulsive strange fixed point inside the disk centered at $27 / 4$ and with radius $81 / 8$. Then, the rounding error in the calculations will determine its orbit. Moreover, we can observe on the parameter space $P_{4}$ (and in $P_{5}$, that has been omitted because of its similitude with $\left.P_{4}\right)$ a little white disk $D_{3}$ on the rim of the left side: it is the region where strange fixed points $e x_{3}(\gamma)$ and $e x_{4}(\gamma)$ are attractive (see Figure 5). Also in parameter planes $P_{i}, i=1,3,4$ a white cardioid at the right side of $D_{2}$, which is the main stability region of the set of 2-periodic orbits.

\section{Dynamical planes}

Finally, by means of the dynamical planes, we will show the qualitative behavior of some different elements of the family (1). We will select the values of the $\gamma$ parameter by using the main conclusions obtained above by analyzing the parameter planes and the stability regions. As in the case of parameter planes, the dynamical planes have been generated
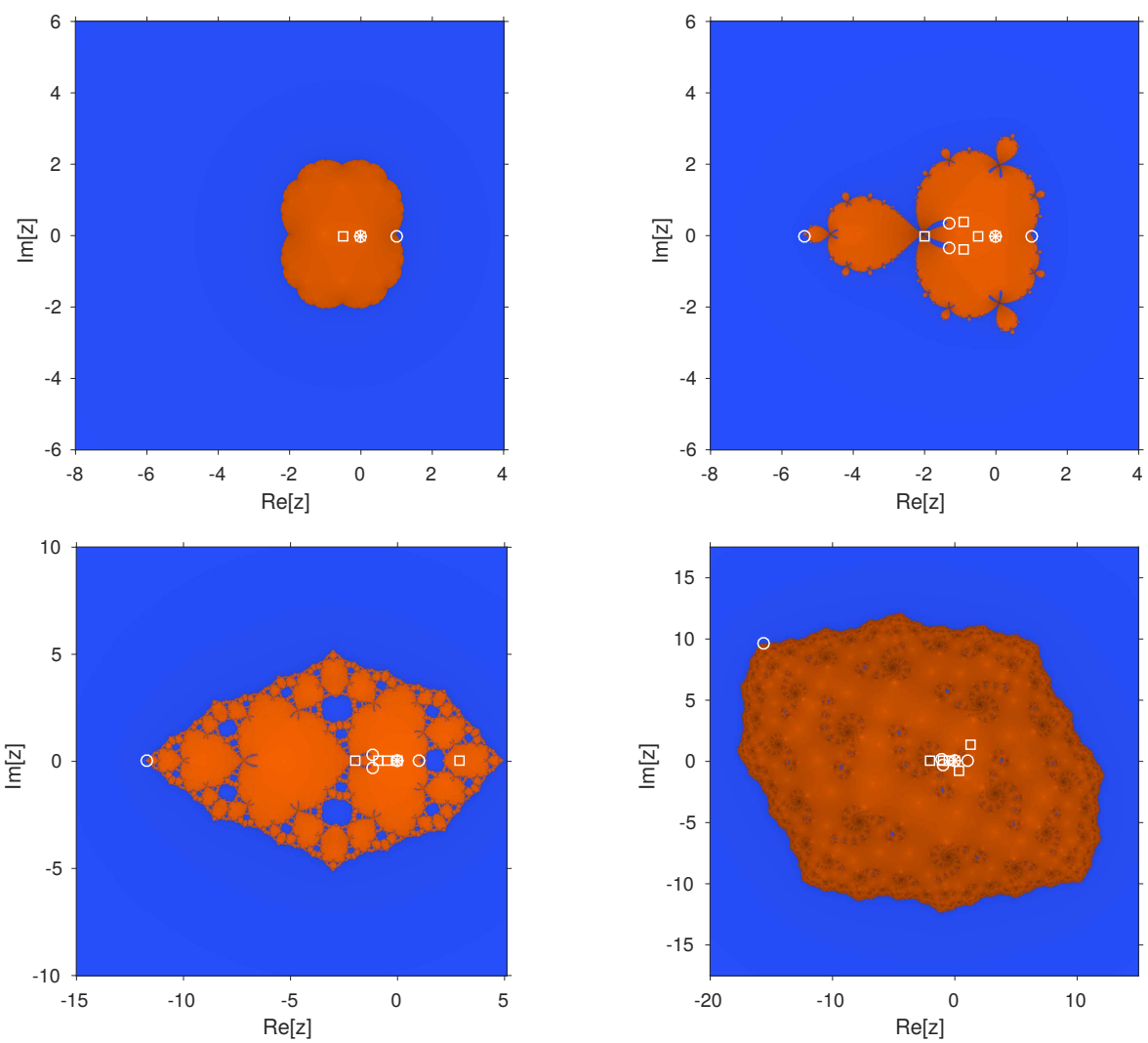

Figure 9: Dynamical planes with stable behavior

by using the routines appearing in [19]. The dynamical plane associated to a value of the $\gamma$ parameter, that is, obtained by iterating an specific method of the family, is generated by using each point of the complex plane as initial estimation (we have used a mesh of $1000 \times 1000$ points). We paint in orange the points whose orbits converge to zero (with a tolerance of $10^{-3}$ ), in blue the points converging to infinity, in green those points whose orbits converge to one of the strange fixed 
points, and in black if it reaches the maximum number of 100 iterations without converging to any of the fixed points. In the figures, the fixed points appear marked as a white circles, the critical points with squares and the attractors with a star. In the parameter spaces we can observe that there are some regions where the corresponding iterative methods have good
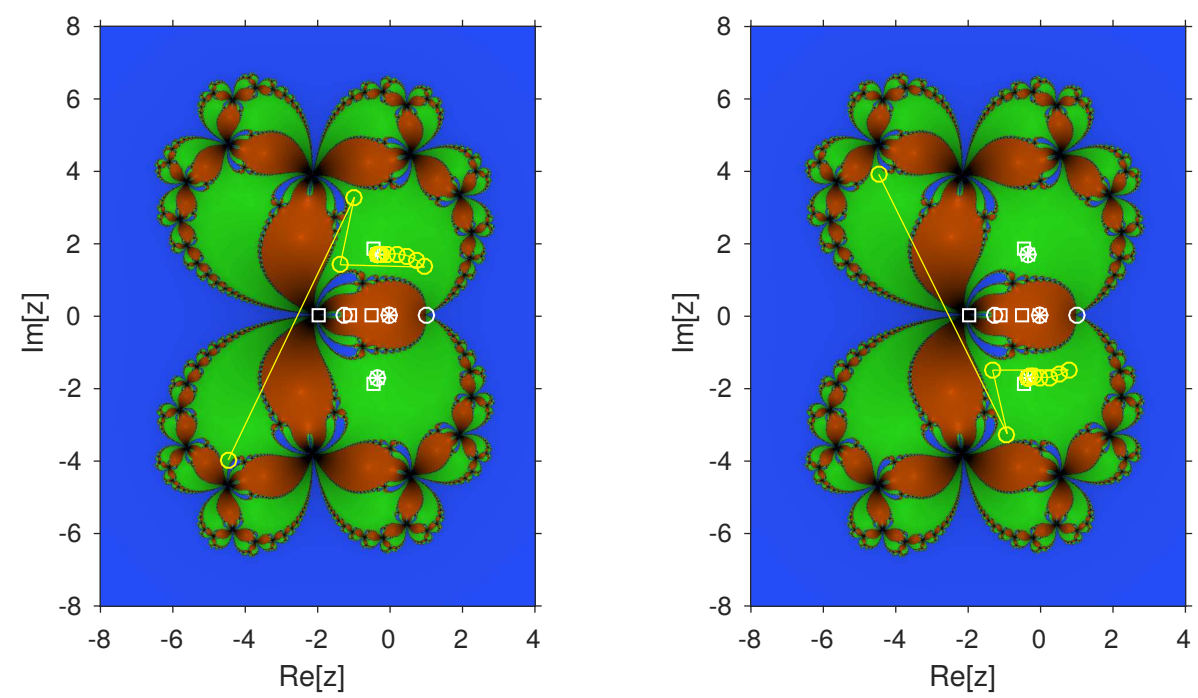

Figure 10: Dynamical planes associated to $\gamma=-2$ with the trajectories (in yellow) converging to $e x_{3}(-2)$ and $e x_{4}(-2)$

numerical behavior in terms of stability. They correspond to values of $\gamma$ painted in red within the disk $|27-4 \gamma|<81 / 2$. In Figure 9 we show different values of the parameter with stable behavior; in particular, we use $\gamma=0$ (Newton's method), $\gamma=1$ (Traub's scheme), $\gamma=4$ (where the family present a convergence of second order), and $\gamma=5.9-4.75 i$. Notice that in the last two cases, the basins of attraction are unconnected.

On the other hand, unstable behavior is found when we choose values of $\gamma$ in the white region of the parameter planes. In Figure 10, the dynamical plane of iterative method corresponding to $\gamma=-2$ is presented, showing the existence of four different basins of attraction: two of them of the attractors 0 and $\infty$, and the other two to the attractors $e x_{3}(-2)$ and $e x_{4}(-2)$. In Figure 11, we represent the dynamical plane corresponding to $\gamma=15$, showing the existence of an attracting 2-periodic orbit, showed by means of yellow lines and circles.

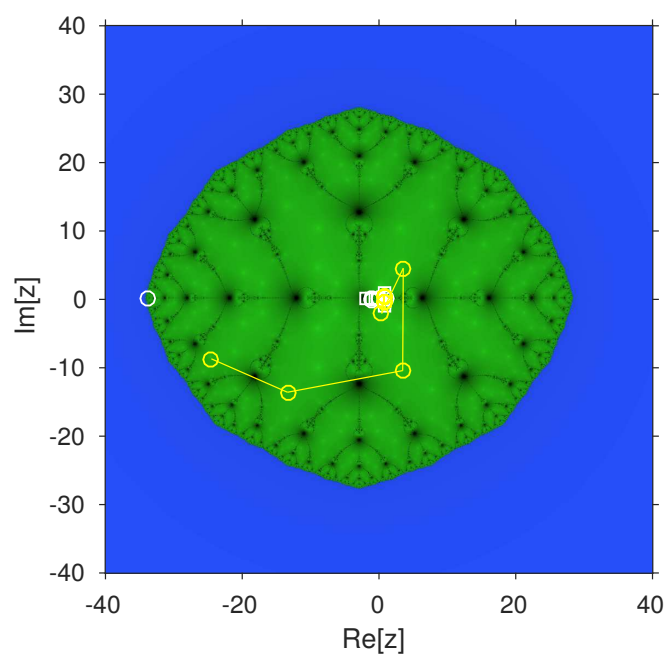

Figure 11: Unstable dynamical plane associated to $\gamma=15$ with the trajectory (in yellow) converging to a two-periodic orbit 


\section{Comparative results}

The comparison among different schemes can be made numerically, by using certain initial estimations, or graphically by means of their basins of attraction. In this section, we show the set of converging initial points for some elements of the family with stable $\left(\gamma_{m}=m^{m}(m-1)^{1-m}, \gamma=8\right.$ and $\left.\gamma=1\right)$ and unstable $(\gamma=-2, \gamma=-3$ and $\gamma=15)$ behavior and Newton's scheme $(\mathrm{Nw})$, the accelerated Newton method (with a damping parameter that is the multiplicity of the root, $\mathrm{aNw}$ ), and Schöeder scheme (Sch) [20], whose iterative expression is

$$
x_{k+1}=x_{k}-\frac{f\left(x_{k}\right) f^{\prime}\left(x_{k}\right)}{\left[f^{\prime}\left(x_{k}\right)\right]^{2}-f\left(x_{k}\right) f^{\prime \prime}\left(x_{k}\right)}, k=0,1,2, \ldots
$$

We apply these procedures on some nonlinear functions coming from applied problems of real life and plot their associate dynamical planes, that have been obtained by using the routines appearing in [19] with a mesh of $800 \times 800$ points, a tolerance of $10^{-3}$ and 200 as maximum number of iterations. In them, we paint in blue color the points whose orbits diverge, in orange, green,... the convergent points to the roots and in black those initial guesses that do not converge with 200 iterations.

Example 1. In the study of the multi-factor effect, the trajectory of an electron in the air gap between two parallel plates is given by

$$
x(t)=x_{0}+\left(v_{0}+e \frac{E_{0}}{m \omega} \sin \left(\omega t_{0}+\alpha\right)\right)\left(t-t_{0}\right)+e \frac{E_{0}}{m \omega^{2}}(\cos (\omega t+\alpha)+\sin (\omega+\alpha)),
$$

where $e$ and $m$ are the charge and the mass of the electron at rest, $x_{0}$ and $v_{0}$ are the position and velocity of the electron at time $t_{0}$ and $E_{0} \sin (\omega t+\alpha)$ is the RF electric field between the plates [21]. We choose the particular parameters in the expression (13) in order to deal with a simpler expression, which is defined as follows:

$$
f_{1}(x)=x+\cos (x)-\frac{\pi}{2} .
$$

The above function has one multiple zero at $x=\frac{\pi}{2}$ of multiplicity three.

From the basins of attraction presented in Figure 12, it can be observed that cases $\gamma_{m}, \gamma=8$ and $\gamma=1$, classified as among the most stable members of the family, have wider and more brilliant basins of attraction of the multiple roots than those of the unstable members $(\gamma=-2, \gamma=-3$ and $\gamma=15$; however, have also better behavior, in terms of speed of convergence (the brighter is the basin, the lower is the number of iterations used to converge) than classical methods, being similar to them in terms of wideness of the basin of attraction.

Example 2. Van der Waals equation of state:

$$
\left(P+\frac{a_{1} n^{2}}{V^{2}}\right)\left(V-n a_{2}\right)=n R T
$$

explains the behavior of a real gas by introducing in the ideal gas equations two parameters, $a_{1}$ and $a_{2}$, specific for each gas. The determination of the volume $V$ of the gas in terms of the remaining parameters requires the solution of a nonlinear equation in $\mathrm{V}$,

$$
P V^{3}-\left(n a_{2} P+n R T\right) V^{2}+a_{1} n^{2} V-a_{1} a_{2} n^{2}=0 .
$$

Given the constants $a_{1}$ and $a_{2}$ of a particular gas, one can find values for $n, P$ and $T$, such that this equation has three simple roots. By using the particular values, we obtain the following nonlinear function

$$
f_{2}(x)=x^{3}-5.22 x^{2}+9.0825 x-5.2675 .
$$

which has three zeros and out of them one is the multiple zero $x=1.75$ of multiplicity 2 , and the other is the simple zero $x=1.72$. However, our desired zero is $x=1.75$.

In Figure 13, we can see that the basin of attraction of the simple root (in green in the figures) is very wide in cases of Newton's, accelerated Newton's method and cases $\gamma_{m}$ and $\gamma=1$. However, the dynamical planes of Schöder's scheme or the stable element of the family corresponding to $\gamma=8$ show almost global convergence. Unstable elements of the class show very bad behavior, as can be observed in the wideness of the black areas of no convergence to the roots. 


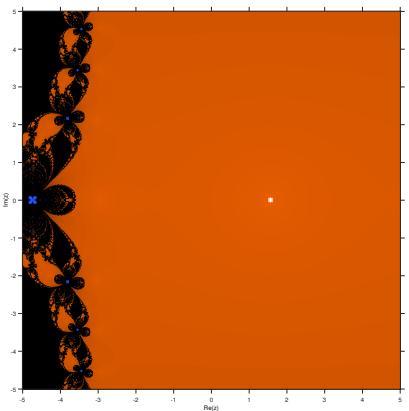

(a) $\mathrm{Nw}$

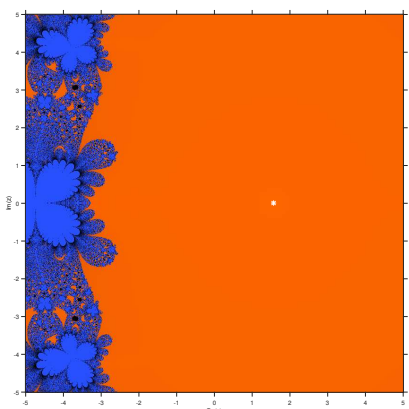

(d) $\gamma_{m}$

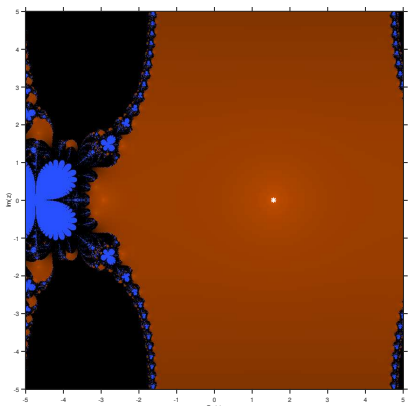

(g) $\gamma=-2$

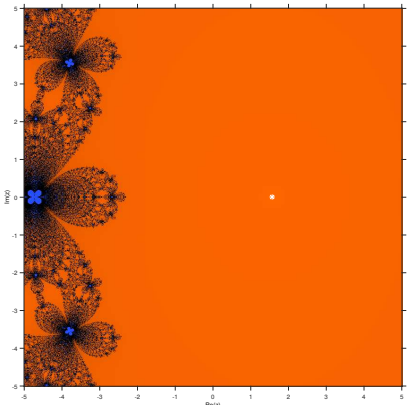

(b) $\mathrm{aNw}$

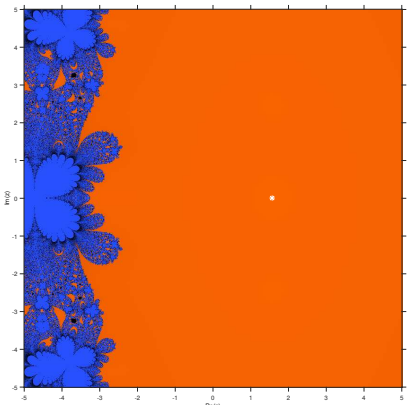

(e) $\gamma=8$

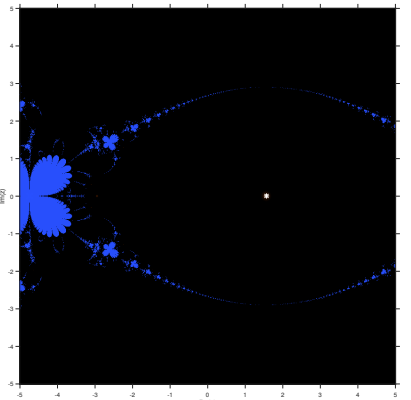

(h) $\gamma=-3$

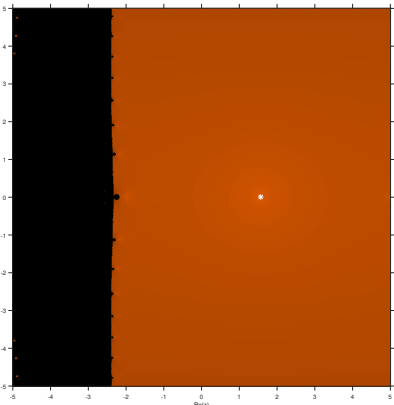

(c) Sch

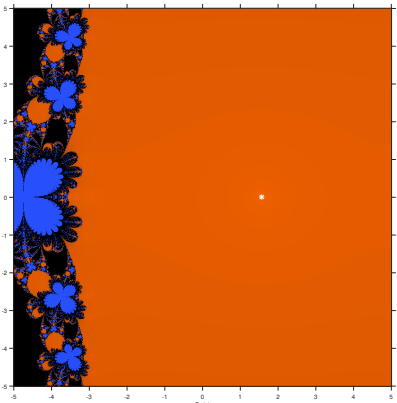

(f) $\gamma=1$

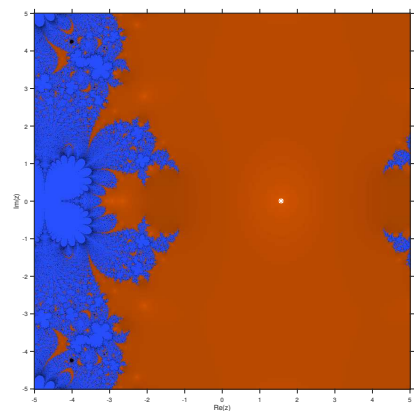

(i) $\gamma=15$

Figure 12: Dynamical planes of the different methods on test function $f_{1}(x)$

Example 3. Let us consider the following nonlinear equation chosen from [22]

$$
f_{3}(x)=\left(\cos \left(\frac{\pi x}{2}\right)-\sqrt{1-x^{2}}+x+1\right)^{3} .
$$

The above function has one multiple zero at $x \approx-0.7285840464448267167123331$ of multiplicity three.

The results appearing in Figure 14 accelerated Newton's method has a similar behavior as the most stable members of the proposed class, that behave much better than Schöder's scheme and those elements corresponding to the set of unstable elements of the class, whose basins of convergence to the multiple root are very small or do not exist.

\section{Conclusions}

In this manuscript, we have focused our attention on the stability analysis of a parametric family of iterative methods (containing the well-known Newton' and Traub's schemes) where the method is applied to functions with multiple roots. It has been demonstrated that the order of convergence depends both on the multiplicity of the roots and the parameter which determine the method. Moreover, the dynamical analysis of the designed family on cubic polynomials with multiple roots gives us important information about its stability, depending on the parameter. It has been proved that it is possible 


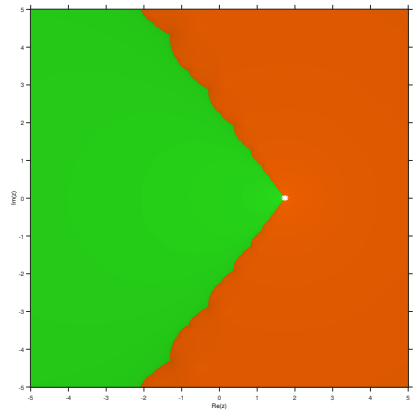

(a) $\mathrm{Nw}$

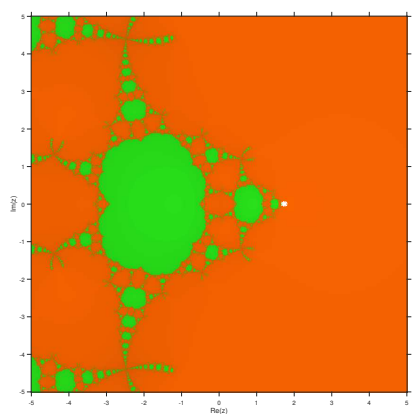

(d) $\gamma_{m}$

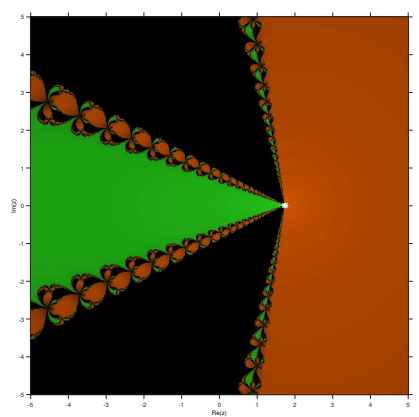

(g) $\gamma=-2$

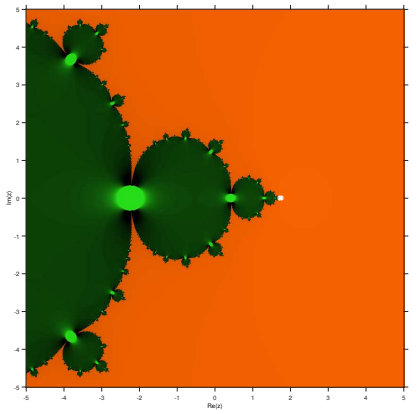

(b) $\mathrm{aNw}$

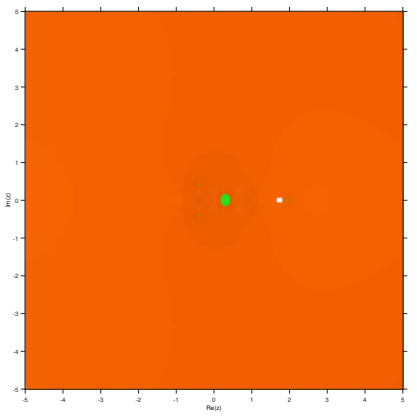

(e) $\gamma=8$

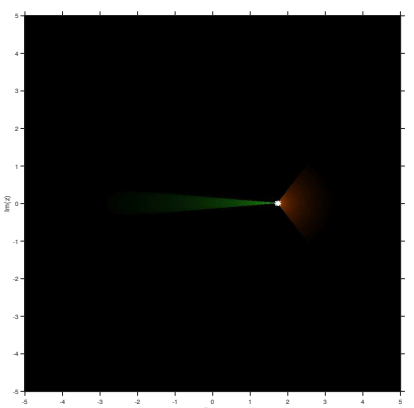

(h) $\gamma=-3$

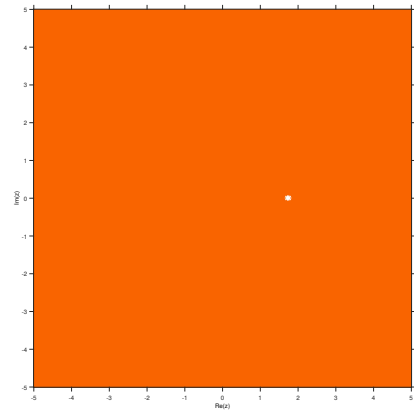

(c) Sch

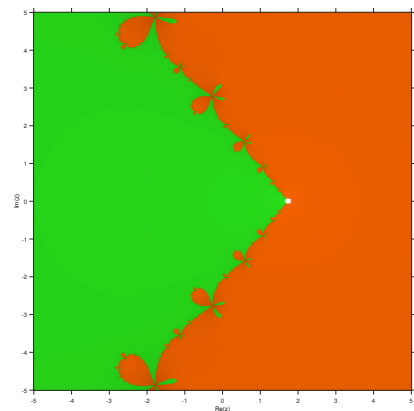

(f) $\gamma=1$

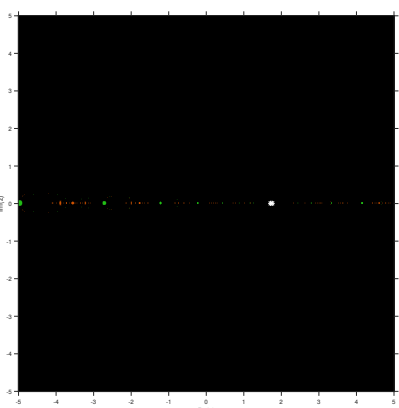

(i) $\gamma=15$

Figure 13: Dynamical planes of the different methods on test function $f_{2}(x)$

to find a complex region of values of $\gamma$, that is a subset of methods of the class, that are able to converge to both roots, multiple (with linear convergence in general and quadratic for $\gamma=4$ ) and simple (with quadratic convergence). From the parameter space, it has been proved that there are many regions with no convergence to the roots of the polynomials, and the existence of periodic orbits of period two has been showed. These members of the family show undesirable numerical behavior. Nevertheless, there are wide regions in parameter space, mainly in the disk of the complex plane centered in $27 / 4$ and with radius $81 / 8$, whose corresponding iterative methods are stable and efficient. These regions include Newton's and Traub's methods, but also many other new stable schemes. These statements have been confirmed by some test made on the nonlinear equations arising from some real-life problems, in which they have shown to be, in some cases, even better than classical methods in terms of stability.

Acknowledgments: The authors thank to the anonymous referees for their valuable comments and for the suggestions that have improved the final version of the paper.

\section{References}

[1] S. Amat, S. Busquier, Advances in Iterative Methods for Nonlinear Equations, Springer, 2016. 


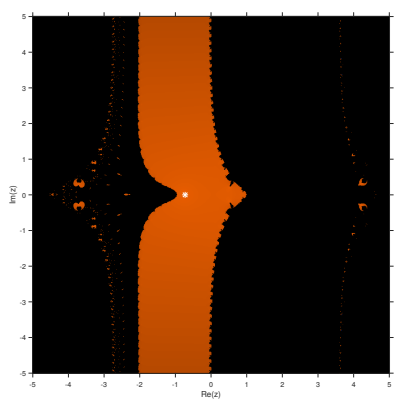

(a) $\mathrm{Nw}$

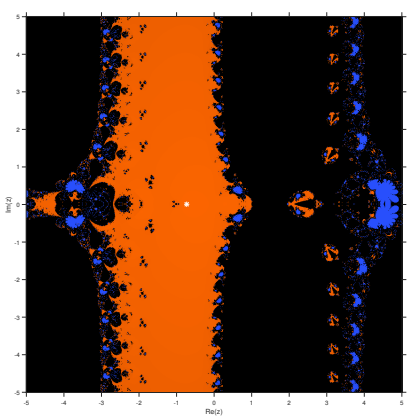

(d) $\gamma_{m}$

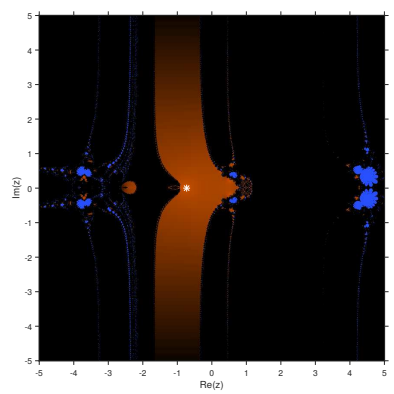

(g) $\gamma=-2$

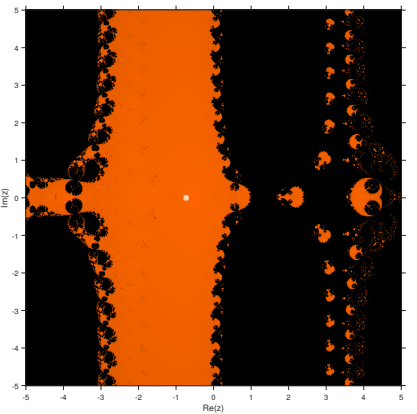

(b) $\mathrm{aNw}$

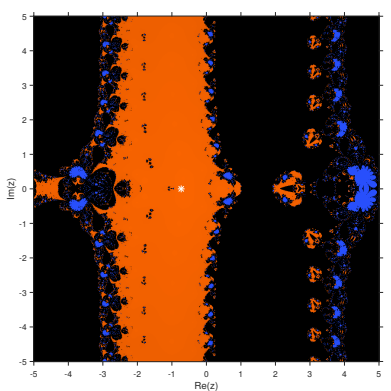

(e) $\gamma=8$

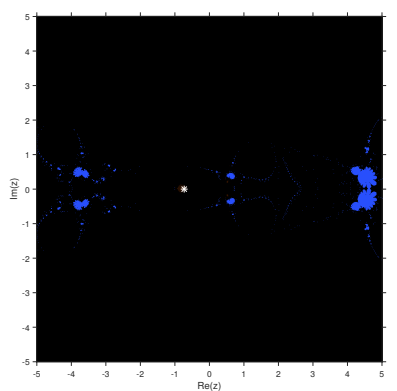

(h) $\gamma=-3$

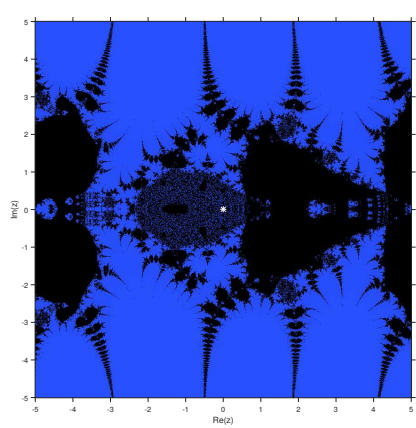

(c) Sch

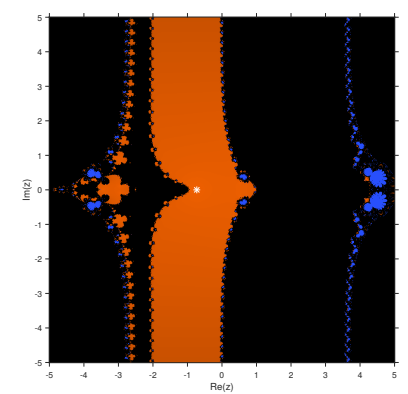

(f) $\gamma=1$

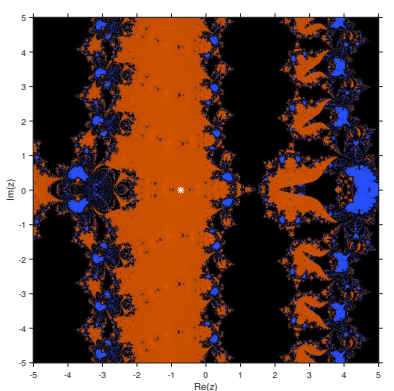

(i) $\gamma=15$

Figure 14: Dynamical planes of the different methods on test function $f_{3}(x)$

[2] M. Petković, B. Neta, L.D. Petković, J. Džunić, Multipoint Methods for Solving Nonlinear Equations, Academic Press, Amsterdam, 2013.

[3] J.F. Traub, Iterative Methods for the Solution of Equations, Chelsea Publishing Company, New York, 1982.

[4] A. Cordero, A. Ferrero, J. R. Torregrosa, Damped Traub's method: convergence and stability, Mathematics and Computers in Simulations 119 (2016) 57-68.

[5] S. Amat, S. Busquier, S. Plaza, Chaotic dynamics of a third-order Newton-type method, J. Math. Anal. Appl. 366 (2010) 24-32.

[6] S. Amat, S. Busquier, S. Plaza, Review of some iterative root-finding methods from a dynamical point of view, Sci. Ser. A: Math. Sci. 10 (2004) 3-35.

[7] S. Artidiello, F. Chicharro, A. Cordero, J.R. Torregrosa, Local convergence and dynamical analysis of a new family of optimal fourth-order iterative methods, Int. J. Comput. Math. 90 (10) (2013) 2049-2060.

[8] I. Argyros, A.A. Magreñán, A study on the local convergence and the dynamics of Chebyshev-Halley-type methods free from second derivative, Numer. Algor. 71(1) (2016) 1-23. 
[9] A. Cordero, J. García-Maimó, J.R. Torregrosa, M.P. Vassileva and P. Vindel, Chaos in King's iterative family, Appl. Math. Lett. 26 (2013) 842-848.

[10] A. Cordero, J.R. Torregrosa, P. Vindel, Dynamics of a family of Chebyshev-Halley type method, Appl. Math. Comput. 219 (2013) 8568-8583.

[11] A.A. Magreñán, Different anomalies in a Jarratt family of iterative root-finding methods, Comput. Appl. Math. 233 (2014) 29-38.

[12] B. Neta, M. Scott, C. Chun, Basins of attraction for several methods to find simple roots of nonlinear equations, Appl. Math. Comput. 218 (2012) 10548-10556.

[13] R. Behl, A. Cordero, S.S. Motsa, J.R. Torregrosa, V. Kanwar, An optimal fourth-order family of methods for multiple roots and its dynamics, Numer. Algor. 71 (2016) 775-796.

[14] Y.H. Geum, Y.I. Kim, B. Neta, A sixth-order family of three-point modified Newton-like multiple-root finders and the dynamics bhind their extraneous fixed points, Appl. Math. Comput. 283 (2016) 120-140.

[15] W.J. Gilbert, The complex dynamics of Newton's method for a double root, Computers Math. Applic. 22(10) (1991) 115-119.

[16] J.M. Gutiérrez, L.J. Hernández, Á.A. Magreñán, M.T. Rivas, Measures of the basins of attracting n-cycles for the relaxed Newton's method, Advances in iterative methods for nonlinear equations, Springer, 2016.

[17] P. Blanchard, The Dynamics of Newton's Method, Proc. Symp. Appl. Math. 49 (1994) 139-154.

[18] P. Blanchard, Complex Analytic Dynamics on the Riemann Sphere, Bull. AMS 11(1) (1984) 85-141.

[19] F. Chicharro, A. Cordero, J.R. Torregrosa, Drawing dynamical and parameter planes of iterative families and methods, Sci. World J. 2013 (2013) Article ID 780153.

[20] E.O. Schröder, On infinitely many algorithms for solving equations, Math. Ann. 2 (1870), 265-317. Traducido por G. W. Stewart, 1992 (disponible en el directorio pub/reports de thales.cs.umd.edu).

[21] Hueso, J.L., Martínez E., Teruel, C.: Determination of multiple roots of nonlinear equations and applications. J. Math. Chem. 53 (2015) 880-892.

[22] R. Behl, A. Cordero, S.S. Motsa, J.R. Torregrosa, V. Kanwar, An optimal fourth-order family of methods for multiple roots and its dynamics. Numer. Algor. 71 (4) (2016) 775-796. 